\title{
Identification and characterization of new plant microRNAs using EST analysis
}

\author{
Bao Hong ZHANG ${ }^{1,2, *}$, Xiao Ping PAN ${ }^{1}$, Qing Lian $W_{A N G}^{2}$, George P. COBB ${ }^{1}$, Todd A. ANDERSON ${ }^{1}$ \\ ${ }^{I}$ The Institute of Environmental and Human Health (TIEHH), and Department of Environmental Toxicology, Texas Tech \\ University, Lubbock, TX 79409-1163, USA \\ ${ }^{2}$ Henan Institute of Science and Technology, Xinxiang 453003, China
}

\begin{abstract}
Seventy-five previously known plant microRNAs (miRNAs) were classified into 14 families according to their gene sequence identity. A total of 18,694 plant expressed sequence tags (EST) were found in the GenBank EST databases by comparing all previously known Arabidopsis miRNAs to GenBank's plant EST databases with BLAST algorithms. After removing the EST sequences with high numbers (more than 2) of mismatched nucleotides, a total of 812 EST contigs were identified. After predicting and scoring the RNA secondary structure of the 812 EST sequences using mFold software, 338 new potential miRNAs were identified in 60 plant species. miRNAs are widespread. Some microRNAs may highly conserve in the plant kingdom, and they may have the same ancestor in very early evolution. There is no nucleotide substitution in most miRNAs among many plant species. Some of the new identified potential miRNAs may be induced and regulated by environmental biotic and abiotic stresses. Some may be preferentially expressed in specific tissues, and are regulated by developmental switching. These findings suggest that EST analysis is a good alternative strategy for identifying new miRNA candidates, their targets, and other genes. A large number of miRNAs exist in different plant species and play important roles in plant developmental switching and plant responses to environmental abiotic and biotic stresses as well as signal transduction. Environmental stresses and developmental switching may be the signals for synthesis and regulation of miRNAs in plants. A model for miRNA induction and expression, and gene regulation by miRNA is hypothesized.
\end{abstract}

Keywords: microRNA, plant, environmental stress, gene regulation, expressed sequence tags.

\section{INTRODUCTION}

MicroRNAs (miRNAs) are a large family of about 2122 nucleotide non-coding RNAs [1-3]. miRNAs play very important roles in posttranscriptional gene regulation by degradation of target mRNAs or by repression of targeted gene translation in animals, plants, and fungi [1-5]. According to the miRNA Registry Database (Release 3.1, April 2004), 899 miRNA genes have been discovered in various organisms [6]. Out of those, 71 miRNA genes have been predicted and identified in plants, of which 43 are from Arabidopsis and 28 from rice (Oryza sativa). After searching the DNA databases (http://www.ncbi.nlm. nih.gov/), we found 4 more miRNA genes discovered in previous reports $[7,8]$; three (microRNA 166a, b and c)

*Correspondence: Bao Hong ZHANG

Tel: +1-806-8854567; Fax: +1-806-8854577;

E-mail: baohong.zhang@ttu.edu from maize (Zea mays) [7], and one (microRNA 166a) from wood tobacco (Nicotiana sylvestris) [8].

Although miRNAs are one of the hottest research topics in biology, the discovery of miRNAs was dominated by chance and serendipity [9]. Currently, most miRNAs were identified by direct cloning of small RNAs $[10,11]$ or by computational strategies [12-17]. Computational strategies suggest that about $1 \%$ of predicted protein-coding genes were miRNAs in metazoan Drosophila [10] and in humans [18-20]. Thus, many miRNA genes still await discovery. Computational strategies provide a useful method to predict miRNA genes and their targets, and have been successfully applied in vertebrates [18-21], insects [22], Arabidopsis and rice [13, 14, 17, 23, 24]. However, many miRNA genes and their targets, especially in plants, still remain undiscovered. The traditional computational approach used was a bit inefficient and certainly not comprehensive, and it is very difficult to demonstrate the expression of these predicted miRNAs using this approach. 
Expressed sequence tags (ESTs) are partial cDNA sequences of expressed genes cloned into a plasmid [25, 26]. EST analysis has proven to be an economically feasible alternative for gene discovery in species lacking a draft genome sequence [26], and many important genes have been found thorough EST analysis [27-29]. As the number of ESTs dramatically increased and became available in databases, they have become a very powerful tool for discovery of new genes and for solving mysteries in biology [29]. As of June 2004, GenBank (National Center for Biotechnology Information, http://www.ncbi.nlm.nih.gov/) contained 22,165,266 entries in its EST database [30]. The greatest number of plant entries are for wheat (Triticum aestivum) $(552,245)$, maize (Zea mays) $(397,515)$, barley (Hordeum vulgare plus subsp. Vulgare) $(356,856)$, soybean (Glycine max) $(334,668)$, rice (Oryza sativa) $(284$, 006), and Arabidopsis thaliana (thale cress) $(258,825)$. This large number of ESTs provides a cost-effective and rapid alternative route toward the discovery and isolation of new genes.

Recently, Jones-Rhoades and Bartel (2004) briefly mentioned that they used Arabidopsis microRNA against an EST database to look for potential evidence of miRNAs in other plant species [14]. Bonnet et al. (2004) also used an EST database to confirm their 91 new potential microRNAs identified by traditional computational strategies [13]. However, they did not do detailed research in this field; they only focused on identifying new miRNAs using a traditional computational strategy. In this paper, based on the analysis of previously known miRNAs, we used EST analysis and DNA database analysis in detail to identify new potential miRNA genes and their targets. Because the ESTs and expressed genes come from the production of true gene expression, our analysis provides more evidence and confidence in the discovery of new potential miRNAs and their targeted genes. Using this strategy, we identified 338 new miRNA candidates in 60 plant species, and 348 genes associated with 348 ESTs targeted by these miRNA candidates. Based on these results, we formed the hypothesis that stress may play an important role in controlling miRNA expression.

\section{MATERIALS AND METHODS \\ Known plant microRNA analysis}

Previously known Arabidopsis (At) and rice (Oryza sativa) (Os) miRNA sequences were obtained from the miRNA Registry Database (Release 3.1, April 2004) [6]; maize $(\mathrm{Zm})$ and tobacco $(\mathrm{Ns})$ miRNA were found in publicly available genome databases by comparing all previously known Arabidopsis miRNA to GenBank's DNA databases with BLAST algorithms by Blastn 2.2.9 (May 1, 2004) [31].

Alignments of known plant miRNA were conducted using the DNAMAN software package (Lynnon Corporation, Quebec, Canada
J7V 9M5). Phylogenetic trees were reconstructed using the same DNAMAN software with standard parameters.

\section{Searching EST databases, carrying out EST analysis, and predicting the secondary structure of EST sequences}

The subgroup of Viridiplantae of the publicly available EST databases was searched using Blastn 2.2.9 (May 1, 2004) [31] by comparing all ESTs to all previously known mature Arabidopsis miRNAs (20-24 base pairs) listed in the miRNA Registry Database (Release 3. 1, April 2004) [6]. Blast parameter settings were as follows: expect 1000; the number of descriptions and alignments were 1000. All Blast results were saved. EST sequences which closely matched $(n / n$, $\mathrm{n}-1 / \mathrm{n}$ and $\mathrm{n}-2 / \mathrm{n}$ nucleotide matches, where $\mathrm{n}$ equals the previously known Arabidopsis miRNA length) the previously known Arabidopsis miRNAs were chosen, and their secondary structures were predicted and generated using the Zucker folding algorithm with mFold $3.1[32,33]$. All mFold outputs including information about the number of structures, free energy $(\Delta \mathrm{G} \mathrm{kcal} / \mathrm{mol})$, miRNA-like helicity, the number of arms per structure, size of helices within arms, and size and symmetry of internal loops within arms were analyzed. The predicted secondary fold-back structures were manually inspected by comparing to the characteristics of previously known miRNAs. The secondary structure of hairpin stem-loop was assigned a score according to the strategies used by Lai et al. (2003) [10]. Briefly, free energy ( $\Delta \mathrm{G} \mathrm{kcal} / \mathrm{mol})$ and miRNA-like helicity for each individual arm were evaluated and assigned a score. Helicity was calculated +1 for each paired helicity, -1 for each one-nucleotide symmetric loop and -2 for each two-nucleotide symmetric loop, and an overall score was calculated as (helical score $+(\operatorname{ABS}(\Delta \mathrm{G}) / 2) / 2$. If there was more than one hairpin stem-loop structure for the ESTs containing the miRNAs, each was scored and the hairpin structure with the highest score was considered the miRNA hairpin stem-loop structure.

Predicted miRNAs and their related information were recorded. Closely related EST sequences were blasted against each other and analyzed. If the ESTs had a high similarity (E value less than e-100), it indicated that these ESTs were created from the same mRNA, and were then considered as one miRNA.

\section{RESULTS}

\section{Plant microRNAs are highly conserved}

According to the miRNA Registry database (Release 3. 1, April 2004) [6], 899 miRNAs have been discovered in various organisms. Out of the 899 genes, 71 miRNA genes have been predicted and identified in plants, of which 43 are from Arabidopsis and 28 from rice. After searching the publicly available DNA databases (http://www.ncbi. nlm.nih.gov/), we found 4 more miRNA genes discovered by Juarez et al. (2004) and McHale and Koning (2004), three from maize [7], and one from wood tobacco (Nicotiana sylvestris) [8].

The four plant species represent both dicots and monocots, two major plant categories. There are different nucleotide identity ratios and related genetic distances among the 75 previously known plant miRNAs (Fig. 1). Some miRNAs are very close to each other, such as miRNA 
Identifying new plant microRNAs using EST analysis

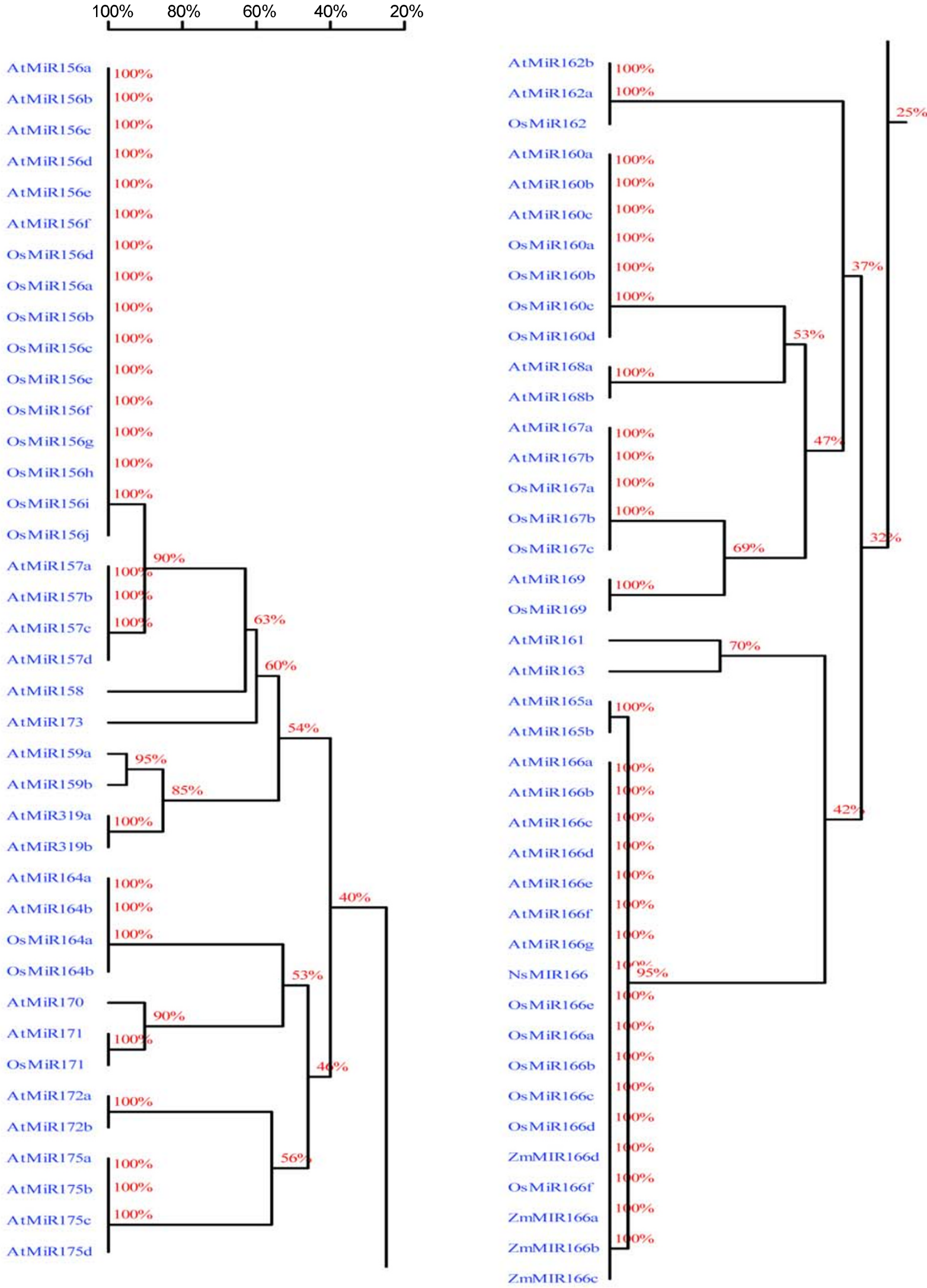

Fig. 1 Nucleotide sequence identity of 75 previously known microRNAs 


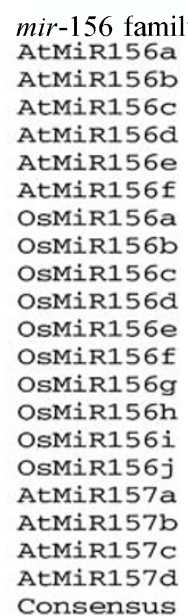

Consensus

mir-158 family

AtMiR 158

mir-159 family

AtMiR159a

AtMiR159b

AtMiR319a

AtMiR319b

Consensus

mir-160 family

AtMiR160a

AtMiR160b

AtMiR160c

OsMiR160a

OsMiR160b

OsMiR160C

OsMiR160d

Consensus
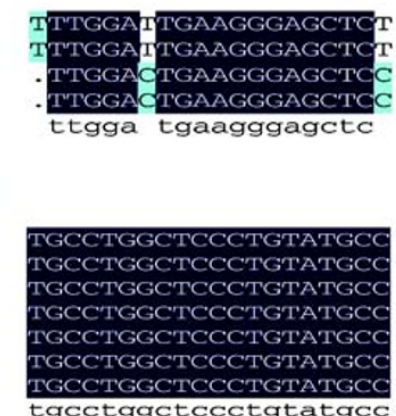

20

mir-161 family

AtMiR161

AtMiR163

Consensus

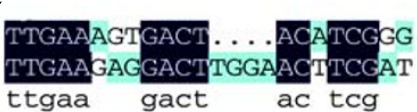

mir-162 family AtMiR162a

AtMiR162b

OsMiR162

Consensus

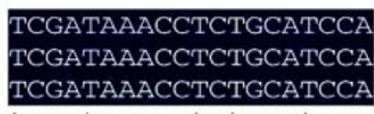

tcgataaacctctgcatcca

mir-164 family

AtMiR164a

AtMiR164b

OsMiR164a

OsMiR164b

Consensus mir-165 family AtMiR165a AtMiR165b AtMiR166a AtMiR166b AtMiR166c AtMiR166d AtMiR166e AtMiR166f AtMiR166g OsMiR166a OsMiR166b OsMiR166C OsMiR166d OsMiR166e OsMiR166f NSMIR166 ZMMIR166a ZMMIR166b ZMMIR166C Consensus

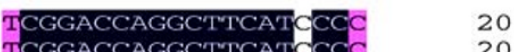
TCGGACCAGGCTTCATTCCC 20 TCGGACCAGGCTTCATTCCC 20 TCGGACCAGGCTTCATTCCC 20 TCGGACCAGGCTTCATTCCC 20 TCGGACCAGGCTTCATYCCO TCGGACCAGGCTTCATICCC 20 TCGGACCAGGCTTCATTCCC 20 TCGGACCAGGCTTCATTCCC 20 TCGGACCAGGCTTCATTCCC 20 TCGGACCAGGCTTCATTCCC TCGGACCAGGCTTCATTCCC 20 .CGGACCAGGCTTCATTCC. 18 TCGGACCAGGCTTCATTCCCे 20 TCGGACCAGGCTTCATTCCC 20 TCGGACCAGGCTTCATTCCO cggaccaggcttcat cC

mir-167 family AtMiR167a

AtMiR167b

OsMiR167a

OsMiR167b

OsMiR167c

AtMiR169

OsMiR169

Consensus

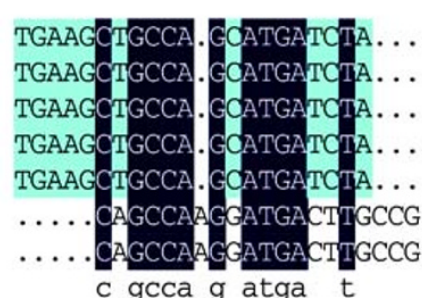

21

21

mir-168 family

AtMiR168a

AtMiR168b

Consensus

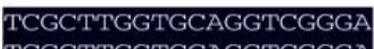

mir-170 family

AtMiR 170

TGATTGAGCCGTGTCAATATC

21

mir-172 family

AtMiR172a

AtMiR172b

Consensus

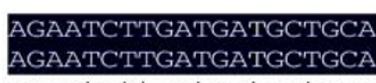

agaatcttgatgatgctgca

20

mir-173 family

AtMiR 173

TTCGCTTGCAGAGAGAAATCAC

22

mir-175 family AtMiR175a

AtMiR175b

AtMiR175c

AtMiR175d

Consensus

Fig. 2 Alignments of 75 previously known plant miRNAs that can be grouped into 14 miRNA families. Arabidopsis $(A t)$ and rice (Oryza sativa) (Os) miRNA are those identified in the miRNA Registry database (Release 3.1, April 2004); maize (Zea mays) ( $\mathrm{Zm}$ ) and tobacco (Nicotiana sylvestris) (Ns) miRNAs are those found in publicly available genome databases by Blastn against all Arabidopsis miRNA genes.

165 and miRNA 166, where there is only 1 nucleotide difference. Others have long genetic distances and low nucleotide identity (Fig. 1), which may be indicative of their relationship and evolution. The 75 miRNAs were classified into 14 miRNA families based on their nucleotide identity (Fig. 2). In each miRNA family, each member 
shares the same or similar gene sequences (Fig. 2). In the miRNAs already reported, only miRNA 166 was found in all four plant species. Fig. 1 and 2 show that these miRNAs from different species are highly conserved, and they share the same DNA sequence. These data suggest that miRNAs may have the same ancestor in very early evolution.

\section{Identifying new plant microRNAs and their targets}

After comparing the gene sequences of 75 previously known miRNAs in different plant species, our results clearly showed that previously known miRNAs are highly conserved (Fig. 1 and 2). In previous reports, several labs have identified some previously known Arabidopsis miRNA homologs in rice $[1,2,11,34]$. Both our results and other reports suggested that different plant species share similar miRNA sequences. Based on these results, in this study, we employed EST analysis to identify new miRNAs in other plant species. Our strategy included the following three steps. First, we searched the EST databases to find ESTs matched with the previously known Arabidopsis miRNAs. Then we predicted the secondary structures of the identified ESTs in the first step using RNA mFold software. Finally, we identified new miRNAs.
First, we searched the plant EST databases using Blastn 2.2.9 (May 1, 2004) [31] against all known Arabidopsis miRNA genes listed in the miRNA Registry database (Release 3.1, April 2004) [6]. A total of 18,694 Blast hits were found in the databases. To avoid a miscount of the total EST sequences, ESTs with n-2, n-3, n-4, and n-5 mismatched nucleotides with previously known miRNA were chosen and paired with the known miRNA sequences. After removing the EST sequences with high numbers (more than 2) of mismatched hits, we obtained 171, 440, and 201 ESTs with 0, 1, and 2 mismatched nucleotides, respectively (Tab. 1 and 2). This provided a total of 812 ESTs for predicting the RNA secondary structures using RNA mfold software.

EST hits differ in EST databases among previously known miRNAs. miRNAs 156 and 157 received the highest number of EST hits, each more than 200 with 0-2 mismatched nucleotides; the 24 and 18 ESTs containing completely conserved sequences were received for miRNAs 156 and 157, respectively. miRNAs 161 and 163 did not receive any EST hits with 0-2 mismatched nucleotides. Although miRNAs 171 and 166 only received 65 and 32 EST hits, respectively; they received the highest number of

Tab. 1 Summary of plant EST blast hits to known plant miRNA sequences

\begin{tabular}{|c|c|c|c|c|c|c|c|c|c|c|c|}
\hline \multirow[t]{2}{*}{ miRNA } & \multirow[t]{2}{*}{ E value } & \multirow[t]{2}{*}{ Blast hits } & \multicolumn{4}{|c|}{$\begin{array}{c}\text { Number of EST with 0-2 mismatched } \\
\text { nuceotides }\end{array}$} & \multicolumn{4}{|c|}{$\begin{array}{l}\text { ESTs for miRNA with } 0-2 \text { mismatched } \\
\text { nuceotides }\end{array}$} & \multirow[t]{2}{*}{ miRNA } \\
\hline & & & 0 & 1 & 2 & Total & 0 & 1 & 2 & Total & \\
\hline 156 & 388 & 1009 & 24 & 185 & & 209 & 22 & 76 & & 98 & 82 \\
\hline 157 & 518 & 1002 & 18 & 94 & 147 & 259 & 13 & 39 & 1 & 53 & 41 \\
\hline 158 & 388 & 984 & 0 & 0 & 13 & 13 & 0 & 0 & 4 & 4 & 4 \\
\hline 159 & 518 & 998 & 11 & 26 & 18 & 55 & 7 & 24 & 4 & 35 & 22 \\
\hline 160 & 518 & 978 & 11 & 36 & & 47 & 8 & 17 & & 25 & 14 \\
\hline 161 & 518 & 984 & 0 & 0 & 0 & 0 & 0 & 0 & 0 & 0 & 0 \\
\hline 162 & 518 & 996 & 6 & 2 & 11 & 19 & 5 & 2 & 1 & 8 & 8 \\
\hline 163 & 906 & 994 & 0 & 0 & 0 & 0 & 0 & 0 & 0 & 0 & 7 \\
\hline 164 & 518 & 998 & 4 & 0 & 6 & 10 & 4 & 0 & 2 & 6 & 6 \\
\hline 165 & 518 & 976 & 1 & 22 & 1 & 24 & 1 & 17 & 0 & 18 & 2 \\
\hline 166 & 518 & 987 & 22 & 4 & 6 & 32 & 17 & 4 & 4 & 25 & 23 \\
\hline 167 & 518 & 984 & 2 & 25 & 1 & 28 & 1 & 18 & 0 & 19 & 15 \\
\hline 168 & 131 & 998 & 14 & 7 & 24 & 45 & 13 & 7 & 24 & 44 & 17 \\
\hline 169 & 518 & 995 & 5 & 9 & 3 & 17 & 5 & 9 & 3 & 17 & 11 \\
\hline 170 & 518 & 822 & 0 & 6 & 43 & 49 & 0 & 6 & 19 & 25 & 21 \\
\hline 171 & 518 & 993 & 40 & 15 & 10 & 65 & 16 & 10 & 5 & 31 & 24 \\
\hline 172 & 518 & 1006 & 6 & 5 & 70 & 81 & 6 & 3 & 39 & 48 & 32 \\
\hline 173 & 647 & 991 & 0 & 0 & 3 & 3 & 0 & 0 & 1 & 1 & 1 \\
\hline 319 & 388 & 999 & 7 & 4 & 23 & 34 & 6 & 3 & 7 & 16 & 14 \\
\hline Total & & 18694 & 171 & 440 & 201 & 812 & 124 & 235 & 105 & 464 & 338 \\
\hline
\end{tabular}


Tab. 2 ESTs with high base pair identity to previously known Arabidopsis miRNAs

\begin{tabular}{|c|c|c|c|}
\hline miRNA & EST without mismatch & EST with $1 \mathrm{nt}$ mismatch & EST with $2 \mathrm{nt}$ mismatch \\
\hline 156 & $\begin{array}{l}\text { CN132665, CF059894, } \\
\text { CF059465, CF059465, } \\
\text { CF039276, CF037245, } \\
\text { CF036982, CF036852, } \\
\text { CF036471, CF036467, } \\
\text { CF036182, CF035885, } \\
\text { CD835236, CB677777, } \\
\text { CB677501, CB674083, } \\
\text { CB653304, CB647907, } \\
\text { CB643501, CA294779, } \\
\text { BI432985, BG300360, } \\
\text { BE807821, AU091537 }\end{array}$ & $\begin{array}{l}\text { CB675101, D41985, CO529327, CO526369, CO525653, CO523543, CO520756, } \\
\text { CO517866, CO414834, AL810223, BP660915, BP657257, CO237265, } \\
\text { CO229128, CO169404, CO121346, CO105970, CO097437, CO097436, } \\
\text { CO092899, CO081765, CO081202, CO051680, CN948866, CN581492, } \\
\text { CN580870, CN578779, CN523533, CN519769, BP056010, BP052650, BP051612, } \\
\text { BP051429, BP051130, BP046017, BP045933, BP044777, BP039258, AV777338, } \\
\text { CK862588, CR285370, CK762549, CK754211, CK753210, CK751799, } \\
\text { CK746364, CK665702, CK441500, CK441500, CK441499, CK441068, } \\
\text { CK294165, CK293650, CK290419, CK286971, CK286411, CK286410, } \\
\text { CK284079, CK284078, CK275093, CK272946, CK262978, CK254420, } \\
\text { CK246692, CK196549, CK119834, CK105751, CK087760, CF836044, } \\
\text { CF833064, CF667730, CF664003, CF663934, CF507028, CF444518, CF229561, } \\
\text { CF035522, BP132966, CD800420, CD481596, CD476205, CD476172, } \\
\text { CD473946, CD472931, CD454302, CD230908, CB970427, CB967554, } \\
\text { CB819319, CB818994, CB632048, CB604551, CB093809, CB093704, } \\
\text { CB091817, BJ576204, BJ571964, BJ568177, BJ567301, BJ566355, CA799494, } \\
\text { CA516324, CA408772, CA408687, CA291432, CA285093, CA263546, } \\
\text { CA254724, CA231663, CA228340, CA210204, CA186028, CA182412, } \\
\text { CA152290, CA148934, CA091338, CA086406, CA082694, CA078294, } \\
\text { CA072223, CA071566, CA032492, CA020690, AU294375, AU293485, } \\
\text { BU833512, BU818933, BU667823, BQ968177, BQ874863, BQ506362, } \\
\text { BQ696284, BQ586808, BJ478354, BQ111685, BJ322639, BJ317100, AV805456, } \\
\text { AV799188, AV788983, AV784285, BM731329, BM731193, BM093898, } \\
\text { BM092276, BM080504, BM064926, BF258419, B1470983, B1425943, } \\
\text { BG947368, BG947367, BG651519, BG650778, BG650023, BG594131, } \\
\text { BG507682, BG447166, BE060620, BG125997, BG040106, BF596468, } \\
\text { BF587970, BF421158, BF273577, BF272491, AU162331, BF097000, BE806824, } \\
\text { BE595949, BE436147, BE329522, AV558614, AV537797, AV524105, } \\
\text { BE057288, AV417559, AW734915, AW679616, A1999403, A1995421, Al738310, } \\
\text { Al726550, CK092224, CB674957 }\end{array}$ & \\
\hline 157 & $\begin{array}{l}\text { CO082782, CO076888, } \\
\text { BP039685, BP030242, } \\
\text { BP029005, BP129308, } \\
\text { BQ916415, BQ119413, } \\
\text { BI971210, BI699573, } \\
\text { BF187371, BE821022, } \\
\text { BE611678, AL368695, } \\
\text { AU089181, BE210632, } \\
\text { AW756919, AW459710 }\end{array}$ & $\begin{array}{l}\text { CN825561, CB330750, CA902638, BJ578566, BJ578544, BJ573618, BJ569965, } \\
\text { BJ568024, BJ563000, BJ558059, BJ556392, CA525602, BM536323, BE435668, } \\
\text { BE433988, AW933950, CO529327, CO526369, CO520756, BP657257, } \\
\text { CO105970, CO097437, CO097436, CO081765, CO081202, CN948866, } \\
\text { CN580870, CN523533, BP046017, CK762856, CK751799, CK745155, } \\
\text { CK294165, CK293650, CK290419, CK286971, CK286411, CK286410, } \\
\text { CK284079, CK284078, CK272946, CK254420, CK246692, CK119834, } \\
\text { CK105751, CK087760, BP132966, CD800420, CD472699, CB970427, } \\
\text { CB819319, CB818994, CB604551, BJ576204, BJ571964, BJ567301, BJ566355, } \\
\text { CA516324, CA408772, CA408687, CA254724, CA228340, CA152290, } \\
\text { CA091338, CA082694, CA072223, CA032492, AU294375, AU293485, } \\
\text { BU818933, BU667823, BQ968177, BQ874863, BQ111685, AV788983, } \\
\text { AV784285, BM731329, BM731193, BF258419, B1425943, BG651519, } \\
\text { BG650778, BG650023, BG447166, BF596468, BF587970, BF273577, BF272491, } \\
\text { AV558614, AV537797, AV524105, AW696064, A1995421, A1738310, } \\
\text { CF059894, }\end{array}$ & \\
\hline 158 & - & - & $\begin{array}{l}\text { BI935773, BI934413, AJ319944, BG139397, } \\
\text { BG139114, BG136021, BU051914, BQ826645, } \\
\text { AI496605, BM309320, BU051914, BQ121631, } \\
\text { BQ826645 }\end{array}$ \\
\hline 159 & $\begin{array}{l}\text { CN007551, CK } 750935, \\
\text { CF210361, CF208653, }\end{array}$ & $\begin{array}{l}\text { CR289947, CK801265, CK801264, CF433018, CF308674, CF303003, CD725199, } \\
\text { CD573488, CD224964, CD224004, CD213304, CD213290, CD204047, }\end{array}$ & \\
\hline
\end{tabular}


Identifying new plant microRNAs using EST analysis

Tab. 2 ESTs with high base pair identity to previously known Arabidopsis miRNAs (continued-1)

\begin{tabular}{|c|c|c|c|}
\hline $\begin{array}{l}\text { miRNA } \\
\end{array}$ & EST without mismatch & EST with $1 \mathrm{nt}$ mismatch & EST with 2 nt mismatch \\
\hline & $\begin{array}{l}\text { CB036045, AU251508, } \\
\text { BU888582, BM893181, } \\
\text { BI265403, AW691937, } \\
\text { AJ388855, }\end{array}$ & $\begin{array}{l}\text { CA731881, CA484819, CA261537, CA236922, CA203816, CA193052, } \\
\text { CA148454, CA079434, AJ475696, BJ456281, BJ448559, BG050158, BE595318, }\end{array}$ & \\
\hline 160 & $\begin{array}{l}\text { CA801322, BU760860, } \\
\text { BG882856, CA002787, } \\
\text { CF330305, CF327976, } \\
\text { CF327535, CF327534, } \\
\text { BF293809, CD445121, } \\
\text { CO484471, }\end{array}$ & $\begin{array}{l}\text { CD480318, BM887596, Al900826, AI794655, BQ401391, CO106264, CO106199, } \\
\text { CO102555, CO095743, BU986263, BJ472971, BJ471957, BJ471544, BJ469735, } \\
\text { BJ469445, AV934494, AV934488, AV933114, AV932125, BF622299, } \\
\text { AW038480, CB643398, CB643394, CA305796, CN520271, CF119506, } \\
\text { CA266669, CA247079, CA209000, CA067925, CF575341, BE494482, } \\
\text { BQ112228, BE921523, CA615738, BQ788860 }\end{array}$ & \\
\hline 161 & - & - & - \\
\hline 162 & $\begin{array}{l}\text { BF003769, BG149136, } \\
\text { B1785837, BQ845158, } \\
\text { BU081804, CF516290, }\end{array}$ & CN894119, CA764295, & CF807240 \\
\hline 163 & - & $\cdot$ & - \\
\hline 164 & $\begin{array}{l}\text { CK936755, CK113235, } \\
\text { CA704421, BU869001 }\end{array}$ & - & $\begin{array}{l}\text { CB009586, BF270853, CD929986, CD899685, } \\
\text { CB651493, CO076098 }\end{array}$ \\
\hline 165 & Св086732 & $\begin{array}{l}\text { Al737566, AJ502524, AW685461, BI893541, BM092635, BM308126, } \\
\text { BQ255147, BQ760548, BQ785760, CA067402, CA067403, CA819984, } \\
\text { CD391899, CD405934, CF626917, CK369135, CN126049, CO531986, } \\
\text { CO521288, AJ771029, AJ770960, AJ770980 }\end{array}$ & - \\
\hline 166 & $\begin{array}{l}\text { Al737566, AJ502524, } \\
\text { AW685461, Bl893541, } \\
\text { BM092635, M308126, } \\
\text { BQ255147, BQ760548, } \\
\text { BQ785760, CA067402, } \\
\text { CA067403, CA819984, } \\
\text { CD391899, CD405934, } \\
\text { CF626917, CK369135, } \\
\text { CN126049, O531986, } \\
\text { CO521288, AJ771029, } \\
\text { AJ770960, AJ770980 }\end{array}$ & BG316028, BJ553847, B1972515, AW694053 & \\
\hline 167 & CK209908, B1095235 & $\begin{array}{l}\text { AU239920, AW318298, AW472145, BG509097, BG510970, BJ556759, } \\
\text { BJ563257, BM892909, BQ629383, BU548271, BU810017, BU886852, } \\
\text { BX928793, CA284319, CA284394, CA287325, CA916400, CD411229, } \\
\text { CF309326, CF322848, CF571260, CF630597, CK209889, CK768366, CN472374 }\end{array}$ & $\mathrm{CD} 003107$ \\
\hline 168 & $\begin{array}{l}\text { H77158, BE659175, } \\
\text { BE661028, AW424354, } \\
\text { CB076866, BU871538, } \\
\text { BU893331, BU889659, } \\
\text { BU888204, BU886509, } \\
\text { BU811772, BU809183, } \\
\text { CF604588, CN901684 }\end{array}$ & BF097936, BE461111, BE461110, CK254942, СK249947, СK246126, CK243936 & $\begin{array}{l}\text { CA003609, CA003589, CB } 667935, \mathrm{CB} 662543, \\
\text { CB655478, CB629782, CA756101, CA173899, } \\
\text { CA156827, CA123434, CF576659, CN145907, } \\
\text { CN133168, CD213423, CD210663, CD209437, } \\
\text { CD209338, CD207772, CD205823, CD205059, } \\
\text { CA826777, BU099153, BG } 842539, \text { BG } 842528\end{array}$ \\
\hline 169 & $\begin{array}{l}\text { BF595231, AW201497, } \\
\text { CF280503, BQ906497, } \\
\text { BU862460, }\end{array}$ & $\begin{array}{l}\text { CA953278, AW596073, BM524615, BM520766, BF598910, BX899561, } \\
\text { BX899553, CD960569, BU865420 }\end{array}$ & CN143986, CA148737, CA148650 \\
\hline 170 & - & CF329292, CD910903, CD441617, CD440809, CA756196, CA009309 & $\begin{array}{l}\text { Al657239, Al770772, AV419737, AW459328, } \\
\text { BE040695, BE365851, BE641457, BG583589, } \\
\text { BG598365, BG653310, BG838164, BJ275219, } \\
\text { BM892213, BM892515, BQ255243, BQ461013, } \\
\text { BX838271, BX839725, BX839739, CA067169, } \\
\text { CA069452, CA120921, CA937914, CB087210, }\end{array}$ \\
\hline
\end{tabular}


Tab. 2 ESTs with high base pair identity to previously known Arabidopsis miRNAs (continued-2)

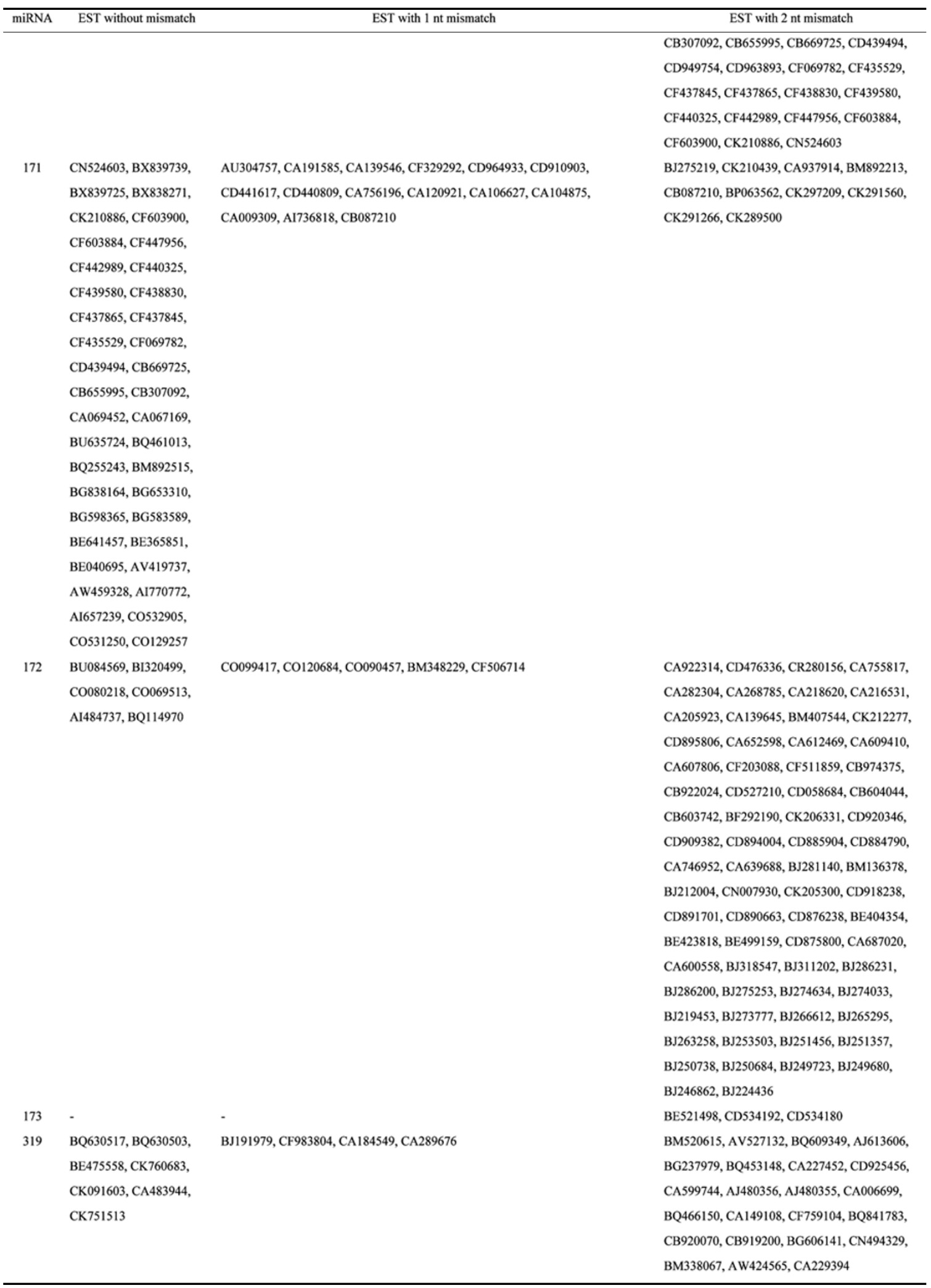


EST hits without nucleotide change. This indicates that the expression of different miRNAs and their number of copies in a plant genome may differ. The higher the number of EST hits, the more copies of the miRNA genes. This also indicates that there is a very high rate of divergence occurring for some miRNAs even if some are highly conserved.

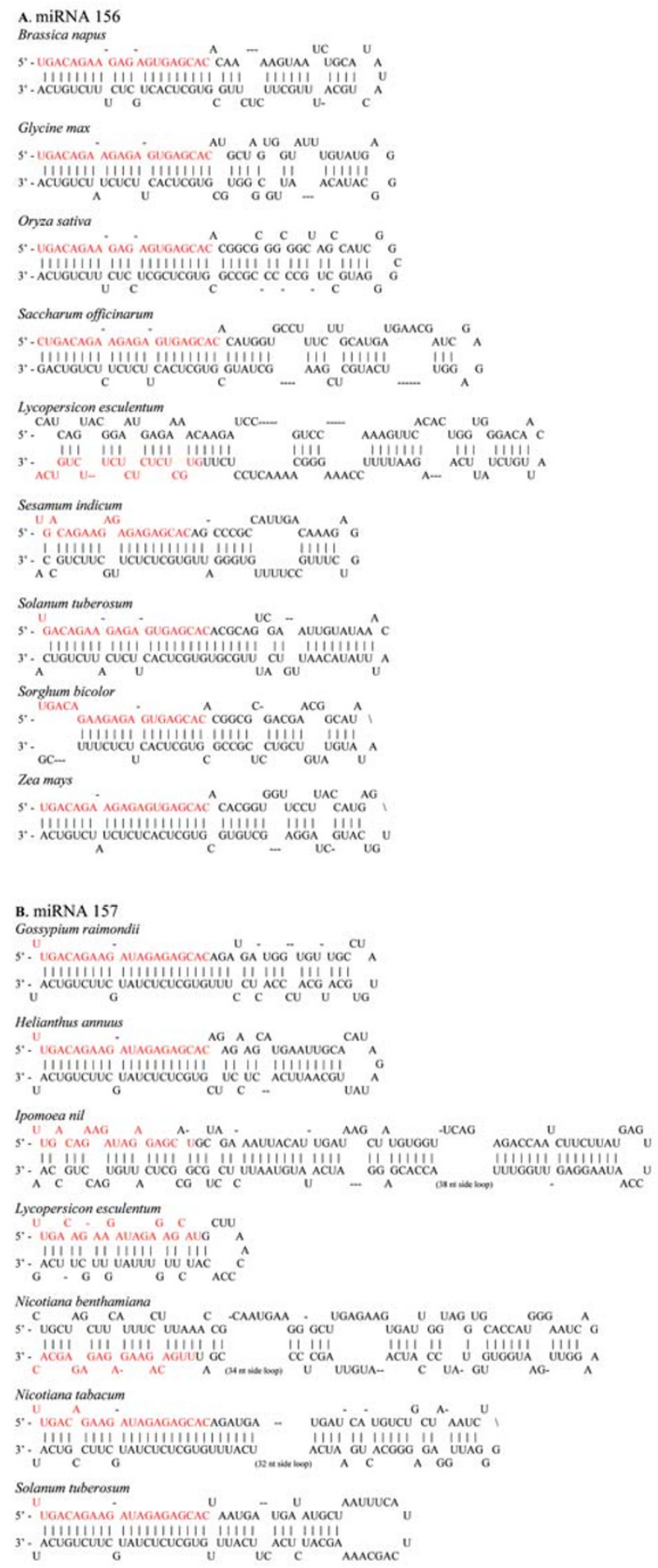

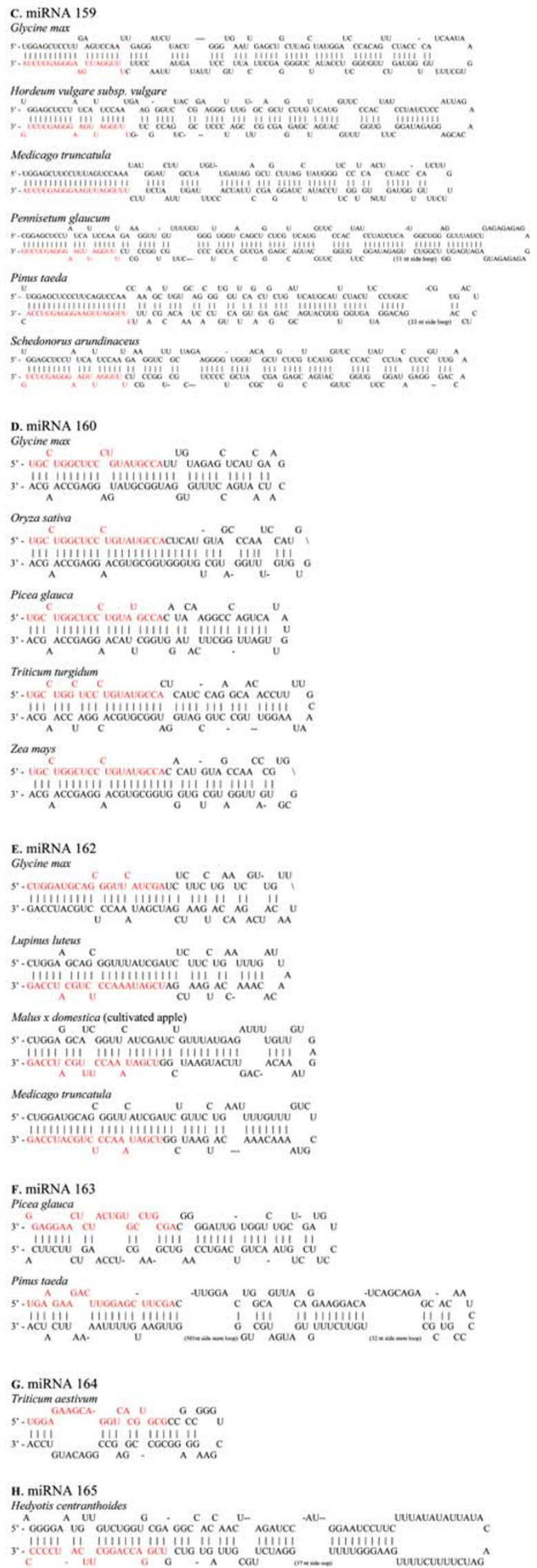




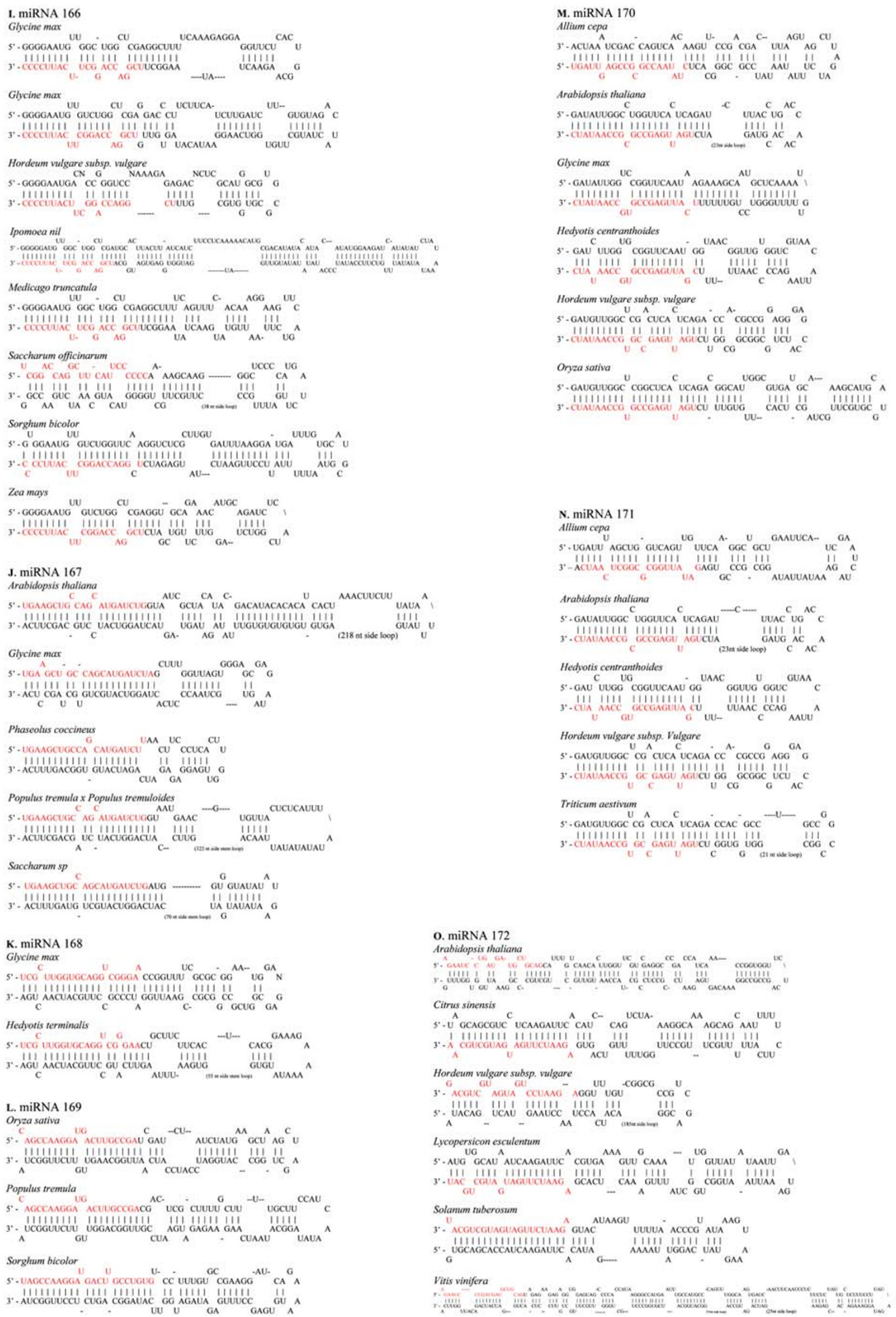




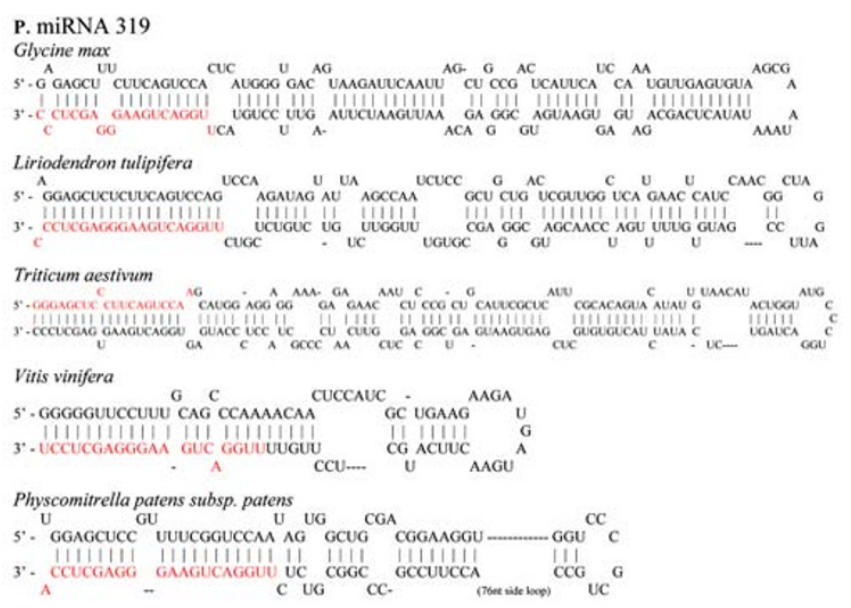

Fig. 3 Examples of the predicated stem-loop structure of newly identified plant miRNA precursors using EST analysis. The miRNA sequences are in grey. The actual size of the precursors may be slightly longer. (A) miRNA 156; (B) miRNA 157; (C) miRNA 159; (D) miRNA 160; (E) miRNA 162; (F) miRNA 163; (G) miRNA 164; (H) miRNA 165; (I) miRNA 166; (J) miRNA 167; (K) miRNA 168; (L) miRNA 169; (M) miRNA 170; (N) miRNA 171; (0) miRNA 172; (P) miRNA 319.

The next step in this study was to predict the secondary structure of all 812 chosen ESTs with 0-2 mismatched nucleotides with previously known Arabidopsis miRNAs, and to identify the new miRNAs and their targeted genes based on the main represented characteristics of miRNAs and their targeted genes. After individually checking and scoring the secondary structure of the 812 chosen ESTs, 464 EST contigs were found to have the patterns of secondary structure conservation resembling those of previously known plant miRNAs (Fig. 3). These 464 ESTs were grouped into 17 families and represented 338 new potential miRNAs in 60 plant species based on their nucleotide sequence similarity as determined by Blastn (Tab. 1 and 3). The other 348 chosen ESTs may be the targets of our newly identified or previously known miRNAs (Tab. 2 and 3).

Out of 171 ESTs without mismatched nucleotides that were compared with previously known Arabidopsis miRNAs, $72.5 \%$ of ESTs (124) were predicted to have the secondary structure of miRNA and were identified as new potential miRNAs. Only $53.4 \%$ and $52.2 \%$ of ESTs had the secondary structure of miRNAs and were identified as new potential miRNAs in the ESTs with 1 or 2 nucleotide substitutions relative to their Arabidopsis miRNA homologs. This also indicated that plant miRNAs are highly conserved.

To avoid designating small RNAs or fragments of other RNAs as miRNAs, Ambros et al. (2003) developed a com- bination of criteria to identify and annotate new miRNAs [35]. The combination of criteria includes both expression criteria and biogenesis criteria. The potential miRNAs identified by EST analysis were highly conserved with the known Arabidopsis miRNAs, and fit the miRNA criteria of secondary struture. Thus, the newly identified potential miRNAs fit the biogenesis criteria well. ESTs are partial cDNA sequences of expressed genes. If this was counted to expression criteria, all new identified potential miRNAs should be true miRNAs. However, to avoid the possibility of artefactual ESTs [36], a Northern blotting method should be employed to test these new potential miRNAs. However, these newly identified gene sequences are still very good miRNA candidates.

In this study, we identified a total of 464 ESTs containing new potential plant miRNAs. Out of those ESTs containing potential miRNAs, 98, the highest number, belong to the miRNA 156 family; 53 belong to the miRNA 157 family; and 48 belong to the miRNA 172 family. miRNAs 156, 157, 159, and 172 are four well studied plant miRNAs. They have important functions in plant development, especially in leaf and flower development. The expression of miRNAs 161 and 163, and their targeted genes are unclear. We did not find any ESTs with 0-2 base substitutions related to these two Arabidopsis miRNAs. However, we found 31 ESTs with 20 matched nucleotides and 4 nucleotide substitutions related to Arabidopsis miRNA 163, and 12 of the 31 ESTs were predicted to have the secondary structure of miRNA, and were identified as new miRNAs. These 12 ESTs expressed 2 miRNAs and belonged to 3 plant species: Picea glauca, Picea sitchensis, and Pinus taeda.

To avoid ESTs created from the same miRNA precursor, ESTs containing the same length hairpin structure were blasted in dbEST against each other. If the ESTs have high similarity, it indicates that these ESTs were created from the same miRNA sequence and they should be considered as one miRNA. After removing the homezygotic ESTs containing the same miRNA, a total of 338 new potential miRNAs were identified in 60 plant species (Fig. 3). The highest number of new potential miRNAs, 45, was found in soybean (Glycine max), followed by rice (Oryza sativa) with 25, sugarcane (Saccharum officinarum) with 25, maize (Zea mays) with 23, and wheat (Triticum aestivum) with 19 (Tab. 4). All of these plant species have more than 200,000 EST sequences in the GenBank's EST databases.

\footnotetext{
Some microRNAs may be induced and regulated by environmental stress; some preferentially express in specific tissues and may be regulated by developmental switching
} 
Tab. 3 New miRNAs identified from plants

\begin{tabular}{|c|c|c|c|c|c|c|c|c|c|c|c|c|c|}
\hline & miRNA & Species & miRNA sequence* & Leng & Precur & & EST & Stra & Folb & miRs & & precur & \\
\hline miRNA & gene & & & (nt) & orhair & i accession & length & nd & ack & Start & End & Start & End \\
\hline family & & & & & $\begin{array}{l}\mathrm{n} \text { leng } \\
(\mathrm{nt})\end{array}$ & number & & & arm & & & & \\
\hline 156 & AtMiR 156m & Arabidopsis thaliana & ugacagaagagagagagcac & 20 & 268 & Al999403 & 498 & + & 3' & 461 & 480 & 213 & 480 \\
\hline & AtMiR 1560 & & & & 245 & AV805456 & 409 & + & 3' & 377 & 396 & 152 & 396 \\
\hline & AtMiR 156p & & & & 47 & AV799188 & 407 & + & 5 , & 326 & 345 & 326 & 372 \\
\hline & AtMiR 156q & & & & 59 & AV558614 & 572 & + & 5 , & 500 & 519 & 500 & 558 \\
\hline & AtMiR 156r & & & & 326 & AV537797 & 607 & + & 5 , & 215 & 234 & 215 & 540 \\
\hline & AtMiR 156s & & & & 218 & Al995421 & 552 & + & 5 , & 223 & 242 & 223 & 440 \\
\hline & BnMiR 156 & Brassica napus & ugacagaagagagugagcac & 20 & 81 & CD835236 & 694 & + & 5 , & 102 & 121 & 102 & 182 \\
\hline & CaMiR 156a & Capsicum anmuum & ugacagaagagagagagcac & 20 & 152 & CA516324 & 508 & - & 3' & 417 & 436 & 285 & 436 \\
\hline & CaMiR 156b & & & & 65 & BM064926 & 474 & - & 5 , & 36 & 55 & 36 & 100 \\
\hline & CsMiR 156a & Citrus sinensis & ugacagaagagagagagcac & 20 & 56 & CK665702 & 605 & - & 3, & 202 & 221 & 166 & 221 \\
\hline & CsMiR 156b & & & & 515 & CF836044 & 785 & + & 3' & 558 & 577 & 63 & 577 \\
\hline & CrMiR 156 & Cycas rumphii & ugacagaagagagagagcac & 20 & 67 & CB091817 & 671 & - & 5 , & 221 & 240 & 221 & 287 \\
\hline & EcMiR 156 & Eschscholzia californica & ugacagaagagagagagcac & 20 & 150 & CD481596 & 691 & - & 3' & 348 & 367 & 218 & 367 \\
\hline & GmMiR 156a & Glycine max & ugacagaagagagugagcac & 20 & 83 & BE807821 & 482 & + & 5 , & 163 & 182 & 163 & 245 \\
\hline & GmMiR 156b & & ugacagaagagagagagcac & 20 & 147 & BM731329 & 421 & - & 5' & 4 & 23 & 4 & 150 \\
\hline & GmMiR 156c & & & & 185 & BM731193 & 504 & - & 5 , & 67 & 86 & 67 & 251 \\
\hline & GmMiR 156d & & & & 76 & BI470983 & 459 & - & 3' & 109 & 128 & 53 & 128 \\
\hline & GmMiR 156e & & & & 55 & BI425943 & 559 & - & 3, & 177 & 196 & 142 & 196 \\
\hline & GmMiR $156 \mathrm{f}$ & & & & 267 & BG651519 & 486 & . & 3' & 373 & 392 & 126 & 392 \\
\hline & GmMiR $156 \mathrm{~g}$ & & & & 209 & BG507682 & 452 & - & 5 , & 77 & 96 & 77 & 285 \\
\hline & GmMiR 156h & & & & 147 & BE806824 & 416 & - & 5 , & 117 & 136 & 117 & 263 \\
\hline & GmMiR 156i & & & & 117 & Aw734915 & 426 & - & 3 , & 174 & 193 & 77 & 193 \\
\hline & HaMiR 156 & Helianthus anmuus & ugacagaagagagagagcac & 20 & 362 & BQ968177 & 575 & - & 5 , & 354 & 373 & 12 & 373 \\
\hline & HsMiR 156 & Hordeum vulgare subsp. spontaneum & ugacagaagagagagagcac & 20 & 178 & BJ478354 & 418 & - & 3' & 325 & 344 & 167 & 344 \\
\hline & HvMiR 156a & Hordeum vulgare subsp. vulgare & ugacagaagagagugagcac & 20 & 394 & BG300360 & 624 & + & 5, & 231 & 250 & 231 & 624 \\
\hline & HvMiR 156b & & ugacagaagagagagagcac & 20 & 86 & CA032492 & 647 & - & 5 , & 523 & 542 & 523 & 608 \\
\hline & HvMiR 156c & & & & 178 & CA020690 & 515 & - & 5 , & 439 & 458 & 281 & 458 \\
\hline & HvMiR 156d & & & & 203 & BF258419 & 902 & - & 3' & 184 & 203 & 1 & 203 \\
\hline & LsMiR 156 & Lactuca sativa & ugacagaagagagagagcac & 20 & 355 & BQ874863 & 654 & - & 5 , & 533 & 552 & 198 & 552 \\
\hline & LjMiR 156 & Lotus corniculatus var. japonicus & ugacagaagagagagagcac & 20 & 47 & BP051612 & 510 & + & 5' & 362 & 381 & 362 & 408 \\
\hline & LeMiR 156a & Lycopersicon esculentum & ugacagaagagagagagcac & 20 & 116 & BF097000 & 482 & - & 3' & 285 & 304 & 189 & 304 \\
\hline & LeMiR 156b & & & & 47 & BG125997 & 589 & - & 5 , & 25 & 44 & 25 & 71 \\
\hline & LeMiR 156c & & & & 263 & BE436147 & 597 & - & 5 , & 21 & 40 & 21 & 283 \\
\hline & MdMiR 156a & Malus $x$ domestica & ugacagaagagagagagcac & 20 & 109 & CN580870 & 505 & - & 5 , & 138 & 157 & 138 & 246 \\
\hline & MdMiR 156b & & & & 79 & CN578779 & 538 & - & 3' & 166 & 185 & 107 & 185 \\
\hline & NbMiR 156a & Nicotiana benthamiana & ugacagaagagagagagcac & 20 & 205 & CK294165 & 812 & + & 5 , & 516 & 535 & 516 & 720 \\
\hline & NbMiR 156b & & & & 169 & CK290419 & 610 & + & 3' & 508 & 527 & 359 & 527 \\
\hline & OsMiR 156m & Oryza sativa & ugacagaagagagugagcac & 20 & 86 & CB677777 & 734 & + & 5 , & 262 & 281 & 262 & 347 \\
\hline & OsMiR 156m & & & & & $\mathrm{CB} 677501$ & 776 & + & 5 , & 260 & 279 & 260 & 345 \\
\hline & OsMiR 156m & & & & & CB653304 & 742 & + & 5 , & 260 & 279 & 260 & 345 \\
\hline & OsMiR 156m & & & & & CB647907 & 722 & + & 5 , & 269 & 288 & 269 & 354 \\
\hline & OsMiR 156m & & & & & CB643501 & 841 & + & 5, & 259 & 278 & 259 & 344 \\
\hline & OsMiR 156m & & & & & AU091537 & 714 & + & 5 , & 10 & 29 & 10 & 95 \\
\hline & OsMiR 156m & & & & & D41985 & 412 & + & 5 , & 286 & 305 & 286 & 371 \\
\hline & OsMiR 156n & & & & 97 & CB674083 & 451 & + & 5 , & 322 & 341 & 322 & 418 \\
\hline & OsMiR 1560 & & ugacagaagagagugagcau & 20 & 179 & CB675101 & 270 & + & 3' & 212 & 231 & 53 & 231 \\
\hline & OsMiR 156p & & ugacagaagagagugagcuc & 20 & 133 & CB674957 & 497 & + & 3' & 333 & 352 & 220 & 352 \\
\hline & OsMiR 156q & & ugacagaacagagugagcac & 20 & 243 & CB632048 & 296 & + & 3, & 237 & 256 & 14 & 256 \\
\hline & OsMiR 156r & & ugacagaagagagagagcac & 20 & 83 & AU162331 & 469 & $\cdot$ & 3' & 398 & 417 & 335 & 417 \\
\hline & PgMiR 156 & Picea glauca & ugacagaagagagagagcac & 20 & 115 & CK441500 & 682 & + & 3' & 627 & 646 & 532 & 646 \\
\hline & PtMiR 156a & Pinus taeda & ugacagaagagagagagcac & 20 & 160 & CF667730 & 787 & - & 3' & 146 & 165 & 6 & 165 \\
\hline & PtMiR 156b & & & & 221 & BQ696284 & 578 & - & 3' & 394 & 413 & 193 & 413 \\
\hline & PpcMiR 156 & Populus balsamifera $x$ Populus deltoids & ugacagaagagagagagcac & 20 & 146 & CN519769 & 797 & - & s' & 336 & 355 & 336 & 481 \\
\hline & PtMiR 156 & Populus tremula & ugacagaagagagagagcac & 20 & 373 & BU818933 & 577 & - & 5 , & 42 & 61 & 42 & 414 \\
\hline & PptMiR 156a & Populus tremula $x$ Populus tremuloides & ugacagaagagagugagcgc & 20 & 46 & CK092224 & 827 & + & 5 , & 414 & 433 & 414 & 459 \\
\hline & PptMiR 156b & & ugacagaagagagagagcac & 20 & 356 & CK087760 & 815 & + & 3' & 455 & 474 & 119 & 474 \\
\hline & PptMiR 156c & & & & 85 & BU833512 & 555 & - & 3' & 284 & 303 & 219 & 303 \\
\hline & PaMiR 156 & Prumus armeniaca & ugacagaagagagagageac & 20 & 114 & CB819319 & 504 & + & 5 ' & 137 & 156 & 137 & 250 \\
\hline & ScMiR 156a & Saccharum officinarum & ugacagaagagagugagcac & 20 & 101 & CA294779 & 738 & + & 5 , & 198 & 217 & 198 & 298 \\
\hline & ScMiR $156 \mathrm{~b}$ & & ugacagaagagagagagcac & 20 & 115 & CA086406 & 691 & + & 3' & 657 & 676 & 462 & 576 \\
\hline & ScMiR 156b & & & & & CA210204 & 735 & - & 3, & 212 & 231 & 113 & 231 \\
\hline & ScMiR 156c & & & & 68 & CA228340 & 637 & - & 3, & 391 & 410 & 343 & 410 \\
\hline & ScMiR 156d & & & & 327 & CA182412 & 563 & - & 5 , & 18 & 37 & 18 & 344 \\
\hline & ScMir $156 \mathrm{e}$ & & & & 70 & CA148934 & 689 & + & 3, & 512 & 531 & 463 & 532 \\
\hline & ScMiR $156 \mathrm{f}$ & & & & 46 & CA091338 & 780 & - & 3, & 163 & 182 & 137 & 182 \\
\hline & ScMiR $156 \mathrm{~g}$ & & & & 403 & CA186028 & 582 & - & 3' & 443 & 462 & 60 & 462 \\
\hline & ScMiR $156 \mathrm{~h}$ & & & & 343 & CA078294 & 1007 & - & 5 , & 28 & 47 & 28 & 370 \\
\hline & ScMiR 156u & & & & 82 & CA072223 & 704 & - & 5 ' & 556 & 575 & 556 & 637 \\
\hline
\end{tabular}


Identifying new plant microRNAs using EST analysis

Tab. 3 New miRNAs identified from plants (continued-1)

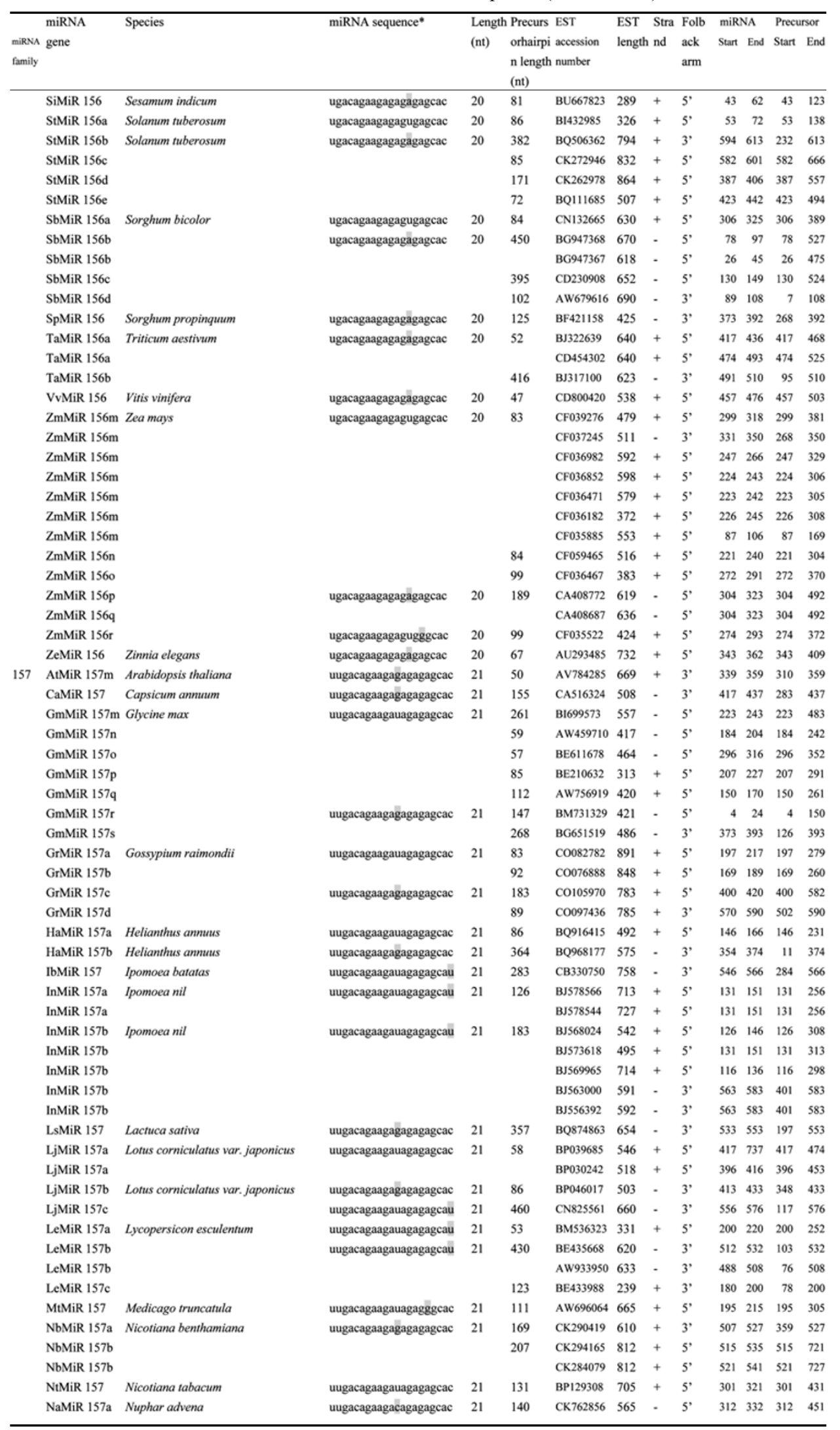


Tab. 3 New miRNAs identified from plants (continued-2)

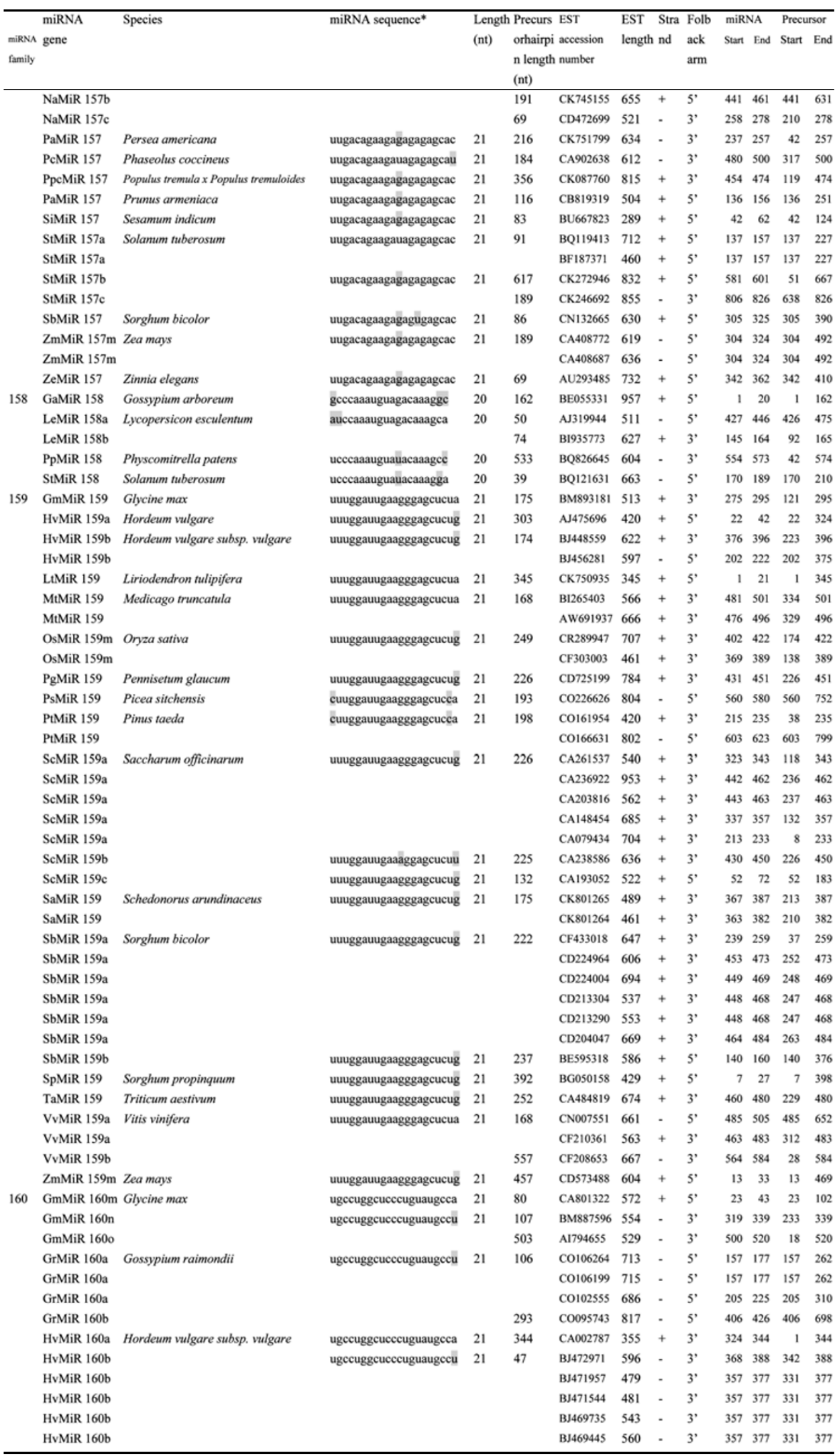


Identifying new plant microRNAs using EST analysis

Tab. 3 New miRNAs identified from plants (continued-3)

\begin{tabular}{|c|c|c|c|c|c|c|c|c|c|c|c|c|c|}
\hline \multirow{3}{*}{$\begin{array}{l}\text { miRNA } \\
\text { family }\end{array}$} & \multirow{3}{*}{$\begin{array}{l}\text { miRNA } \\
\text { gene }\end{array}$} & \multirow[t]{3}{*}{ Species } & \multirow[t]{3}{*}{ miRNA sequence* } & \multicolumn{3}{|c|}{ Length Precurs EST } & \multirow{3}{*}{$\begin{array}{l}\text { EST } \\
\text { lengt }\end{array}$} & \multirow{3}{*}{$\begin{array}{l}\text { Stra } \\
\text { nd }\end{array}$} & Folb & $\operatorname{miRN}$ & & Precur & \\
\hline & & & & (nt) & orhai & accession & & & ack & Start & End & Start & End \\
\hline & & & & & $\begin{array}{l}\mathrm{n} \text { len } \\
(\mathrm{nt})\end{array}$ & number & & & arm & & & & \\
\hline & HvMiR 160b & & & & & AV934494 & 482 & - & $3^{\prime}$ & 358 & 378 & 331 & 378 \\
\hline & HvMiR 160b & & & & & AV934488 & 412 & - & $3^{\prime}$ & 329 & 349 & 333 & 349 \\
\hline & HvMiR 160b & & & & & AV933114 & 608 & - & $3^{\prime}$ & 321 & 341 & 295 & 341 \\
\hline & HvMiR 160b & & & & & AV932125 & 479 & - & 3 & 357 & 377 & 331 & 377 \\
\hline & OsMiR 160m & Oryza sativa & ugccuggcucccuguaugcca & 21 & 82 & CF33030s & 650 & + & 5 ' & 248 & 268 & 248 & 329 \\
\hline & OsMiR 160m & & & & & CF327976 & 627 & + & 5 ' & 254 & 274 & 254 & 335 \\
\hline & OsMiR 160n & & & & 131 & CF327534 & 574 & + & 5 ' & 402 & 422 & 402 & 532 \\
\hline & OsMiR 160n & & & & & CF327535 & 536 & - & 3 & 221 & 241 & 112 & 241 \\
\hline & PgMiR 160 & Picea glauca & ugccuggcucccuguaugeca & 21 & 80 & CO484471 & 770 & + & 5 , & 269 & 289 & 269 & 348 \\
\hline & ScMiR 160 & Secale cereale & ugecuggcucccuguaugecu & 21 & 180 & BE494482 & 556 & - & $3^{\prime}$ & 357 & 377 & 198 & 377 \\
\hline & TtMiR 160a & Triticum turgidum & ugccuggcucccuguaugeca & 21 & 87 & BF293809 & 539 & + & 5 & 259 & 279 & 259 & 345 \\
\hline & TtMiR $160 \mathrm{~b}$ & & ugecuggcucccuguaugecu & 21 & 218 & BQ788860 & 620 & - & $3^{\prime}$ & 264 & 284 & 67 & 284 \\
\hline & ZmMiR 160m & Zea mays & ugecuggcueccuguaugeca & 21 & 81 & CD445121 & 648 & + & 5 , & 285 & 305 & 285 & 365 \\
\hline 162 & GmMiR $162 \mathrm{~m}$ & Glycine max & ucgauaaaccucugcauccag & 21 & 367 & BU081804 & 557 & + & 5 ' & 45 & 65 & 45 & 411 \\
\hline & GmMiR 162n & & ucgaugaaccgcugcauccag & 21 & 84 & CF807240 & 378 & - & 5 & 300 & 320 & 300 & 383 \\
\hline & LsMiR 162 & Lactuca sativa & ucgauaaaccucugcauccag & 21 & 89 & BQ845158 & 367 & + & 5 ' & 240 & 260 & 240 & 328 \\
\hline & LIMiR 162 & Lupimus luteus & ucgauaaaccucugcauccag & 21 & 80 & BG149136 & 442 & + & 3 & 274 & 294 & 215 & 294 \\
\hline & LmMiR 162 & Malus $x$ domestica (cultivated apple) & ucgauaaaccuuugcauccag & 21 & 88 & CN894119 & 327 & + & $3^{\prime}$ & 173 & 193 & 106 & 193 \\
\hline & MtMiR 162 & Medicago truncatula & ucgauaaaccucugcauccag & 21 & 87 & BF003769 & 478 & + & $3^{\prime}$ & 221 & 241 & 155 & 241 \\
\hline & OsMiR 162m & Oryza sativa & ucgauaagccucugcauccag & 21 & 114 & CA764295 & 896 & + & $3^{\prime}$ & 425 & 445 & 332 & 445 \\
\hline & VvMiR 162 & Vitis vinifera & ucgauaaaccucugcauccag & 21 & 85 & CF516290 & 433 & + & 3 & 368 & 388 & 304 & 388 \\
\hline 163 & OsMiR 163m & Oryza sativa & uugaagaggacuuggaacguuc & 21 & 47 & CA763268 & 688 & - & 3 & 621 & 641 & 595 & 641 \\
\hline & PpMiR 163 & Picea glauca & ugaagaagacuuggagcuucga & 22 & 74 & $\mathrm{CO} 236262$ & 723 & - & 3 & 654 & 675 & 602 & 675 \\
\hline & PpMiR 163 & & & & 92 & CO254401 & 890 & - & $3^{\prime}$ & 691 & 712 & 621 & 712 \\
\hline & PsMiR 163 & Picea sitchensis & ugaagaagacuuggagcuucga & 22 & 93 & CO222007 & 839 & - & 3 & 695 & 716 & 624 & 716 \\
\hline & PtMiR 163a & Pimus taeda & ugaagaagacuuggagcuucga & 22 & 93 & CO165688 & 908 & - & 3 & 565 & 586 & 494 & 586 \\
\hline & PtMiR 163a & & & & & CO157878 & 798 & - & $3^{\prime}$ & 573 & 594 & 502 & 594 \\
\hline & PtMiR 163a & & & & & CO163746 & 844 & - & 3 & 648 & 669 & 578 & 669 \\
\hline & PtMiR 163a & & & & & CO163668 & 831 & - & $3^{\prime}$ & 414 & 435 & 343 & 435 \\
\hline & PtMiR 163a & & & & & CO157806 & 707 & - & $3^{\prime}$ & 293 & 314 & 222 & 314 \\
\hline & PtMiR 163a & & & & & CF389355 & 622 & - & 3 & 302 & 323 & 231 & 323 \\
\hline & PtMiR 163a & & & & & CF669738 & 784 & + & 3 & 351 & 372 & 307 & 372 \\
\hline & PtMiR 163b & & & 22 & 650 & CO160078 & 735 & + & 5 & 25 & 46 & 25 & 674 \\
\hline & PtMiR 163b & & & & & CO158895 & 772 & + & 5 ' & 101 & 122 & 101 & 750 \\
\hline & PpbMiR 163 & Populis balsamifera $\times$ Populis deltoides & augaagaggaguuggaacuua & 21 & 100 & CN524551 & 654 & + & 3 & 253 & 273 & 174 & 273 \\
\hline 164 & CsMiR 164 & Citrus sinensis & uggagaagcagggcacgugea & 21 & 168 & CK936755 & 674 & + & 5 & 153 & 173 & 153 & 320 \\
\hline & GaMiR 164 & Gossypium arboreum & uggagaagcagggcacguaaa & 21 & 110 & BF270853 & 579 & - & $3^{\prime}$ & 94 & 111 & 1 & 111 \\
\hline & InMiR 164 & Ipomoea nil & cggagaagcaggucacgugeg & 21 & 87 & BJ577535 & 704 & & $3^{\prime}$ & 502 & 522 & 436 & 522 \\
\hline & PLMiR 164 & Populus balsamifera subsp. trichocarpa & uggagaagcagggcacgugea & 21 & 137 & BU869001 & 621 & + & 5 & 16 & 36 & 16 & 152 \\
\hline & PIMiR 164 & & & & & CK113235 & 537 & + & 5 ' & 159 & 179 & 159 & 294 \\
\hline & TaMiR 164a & Triticum aestivum & uggagaagcaggucacgugca & 21 & 60 & CD899685 & 736 & + & 5 & 615 & 635 & 615 & 674 \\
\hline & TaMiR 164b & & uggagaagcagggcacgugca & 21 & 152 & CA704421 & 476 & + & 5 ' & 10 & 30 & 10 & 161 \\
\hline & TaMiR 164b & & & & & CD929986 & 654 & - & 3 & 171 & 191 & 134 & 191 \\
\hline 165 & HeMiR 165 & Hedyotis centranthoides & ucggaccaggcuucauccece & 21 & 165 & СВ086732 & 632 & + & $3^{\prime}$ & 224 & 244 & 80 & 244 \\
\hline & SbMiR 165 & Sorghum bicolor & ccggaccaggcuucaucccaa & 21 & 426 & CN140010 & 750 & - & $3^{\prime}$ & 726 & 746 & 301 & 726 \\
\hline 166 & GmMiR 166m & Glycine max & ucggaccaggcuucauuccec & 21 & 128 & CD405934 & 400 & - & 5 ' & 112 & 132 & 112 & 239 \\
\hline & GmMiR 166m & & & & & CD391899 & 338 & - & 5 ' & 129 & 149 & 129 & 256 \\
\hline & GmMiR 166m & & & & & CA819984 & 385 & + & 3 & 241 & 261 & 133 & 261 \\
\hline & GmMiR 166n & & & & 409 & BQ785760 & 488 & + & 5 & 47 & 67 & 47 & 455 \\
\hline & GmMiR 1660 & & & & 85 & BM308126 & 468 & + & $3^{\prime}$ & 440 & 460 & 375 & 460 \\
\hline & GmMiR 166p & & & & 106 & BI893541 & 517 & + & $3^{\prime}$ & 125 & 145 & 39 & 145 \\
\hline & GmMiR 166q & & ucggaccaggcuucauucceg & 21 & 156 & B1972515 & 573 & + & 3 & 306 & 326 & 171 & 326 \\
\hline & GmMiR 166r & & ucggaccaggcuucauucccu & 21 & 136 & BG316028 & 415 & + & 3 ' & 238 & 258 & 123 & 258 \\
\hline & HvMiR 166 & Hordeum vulgare subsp. vulgare & ucggaccaggcuucauuccec & 21 & 78 & BQ760548 & 433 & + & $3^{\prime}$ & 136 & 156 & 79 & 156 \\
\hline & InMiR 166 & Ipomoea nil & ucggaccaggcuucauuccuc & 21 & 169 & BJ553847 & 485 & + & $3^{\prime}$ & 310 & 330 & 162 & 330 \\
\hline & MtMiR 166a & Medicago truncatula & ucggaccaggcuucauuccec & 21 & 96 & AJ502524 & 411 & + & 3 & 319 & 339 & 244 & 339 \\
\hline & MtMiR 166a & & & & 94 & BQ255147 & 607 & + & $3^{\prime}$ & 167 & 187 & 94 & 187 \\
\hline & MtMiR 166a & & & & 97 & AW685461 & 568 & + & 3 & 220 & 240 & 144 & 240 \\
\hline & MtMiR 166a & & & & 94 & Al737566 & 304 & + & $3^{\prime}$ & 133 & 153 & 60 & 153 \\
\hline & MtMiR 166b & & ucggaccaggcuucauuccuc & 21 & 171 & AW694053 & 393 & + & 3, & 371 & 391 & 221 & 391 \\
\hline & OsMiR 166m & Oryza sativa & ucggaccaggcuucaaucccu & 21 & 97 & CA760464 & 684 & - & 5 ' & 442 & 462 & 442 & 538 \\
\hline & PgMiR 166 & Picea glauca & ucggaccaggcuucauuccuu & 21 & 74 & C0476713 & 810 & - & 5 ' & 155 & 175 & 155 & 228 \\
\hline & SoMiR 166 & Saccharum officinarum & ucggaccaggeuucauuccec & 21 & 127 & CA067403 & 732 & + & 5 ' & 44 & 64 & 44 & 170 \\
\hline & SoMiR 166 & & & & 131 & CA067402 & 1098 & + & 5 & 44 & 64 & 44 & 172 \\
\hline & SbMiR 166 & Sorghum bicolor & ucggaccaggcuucauuccec & 21 & 108 & CN126049 & 707 & + & $3^{\prime}$ & 157 & 177 & 70 & 177 \\
\hline & TaMiR 166 & Triticum aestivum & ceggaccaggcuucauuccea & 21 & 111 & CK204430 & 801 & - & $3^{\prime}$ & 168 & 188 & 78 & 188 \\
\hline & TaMiR 166 & & & & & CK204088 & 823 & - & $3^{\prime}$ & 167 & 187 & 77 & 187 \\
\hline & ZmMiR 166m & Zea mays & ucggaccaggcuucauuccec & 21 & 87 & CK369135 & 655 & + & 3 ' & 163 & 183 & 97 & 183 \\
\hline
\end{tabular}


Tab. 3 New miRNAs identified from plants (continued-4)

\begin{tabular}{|c|c|c|c|c|c|c|c|c|c|c|c|c|c|}
\hline \multirow{3}{*}{$\begin{array}{l}\text { miRNA } \\
\text { family }\end{array}$} & \multirow{3}{*}{$\begin{array}{l}\text { miRNA } \\
\text { gene }\end{array}$} & \multirow[t]{3}{*}{ Species } & \multirow[t]{3}{*}{ miRNA sequence* } & \multicolumn{2}{|c|}{ Length Precurs } & EST & \multirow{3}{*}{$\begin{array}{l}\text { EST } \\
\text { length }\end{array}$} & Stra & Folb & $\operatorname{miRN}$ & & Precu & \\
\hline & & & & (nt) & orhair & accession & & nd & ack & Start & End & Start & End \\
\hline & & & & & $\mathrm{n}$ leng & number & & & arm & & & & \\
\hline & ZmMiR 166n & & & & 78 & $\cos 31986$ & 680 & + & $3^{\prime}$ & 160 & 180 & 103 & 180 \\
\hline & ZmMiR 1660 & & & & 78 & $\cos 21288$ & 766 & + & $3^{\prime}$ & 161 & 181 & 104 & 181 \\
\hline & ZmMiR 166p & & & & 457 & CF626917 & 661 & - & $3^{\prime}$ & 622 & 642 & 186 & 642 \\
\hline 167 & AtMiR 167m & Arabidopsis thaliana & ugaagcugccagcaugaucug & 21 & 342 & AU239920 & 619 & + & 5 ' & 193 & 213 & 193 & 534 \\
\hline & GmMiR $167 \mathrm{~m}$ & Glycine max & ugaagcugecagcaugaucua & 21 & 82 & B1095235 & 554 & + & 5 , & 405 & 425 & 405 & 486 \\
\hline & GmMiR $167 \mathrm{~m}$ & & & & 64 & CD411229 & 586 & - & $3^{\prime}$ & 496 & 516 & 453 & 516 \\
\hline & GmMiR 167n & & & & 64 & BU548271 & 462 & - & $3^{\prime}$ & 209 & 229 & 166 & 229 \\
\hline & GmMiR 167n & & & & & BM892909 & 489 & + & 5 ' & 124 & 144 & 124 & 187 \\
\hline & GmMiR 167n & & & & & BG509097 & 498 & + & 5 ' & 227 & 247 & 227 & 290 \\
\hline & GmMiR 167o & & ugaagcugecagcaugaucug & 21 & 109 & BQ629383 & 549 & + & $3^{\prime}$ & 222 & 242 & 134 & 242 \\
\hline & InMiR 167 & Ipomoea nil & ugaagcugccagcaugaucug & 21 & 271 & BJ563257 & 578 & + & 5 ' & 265 & 285 & 265 & 535 \\
\hline & OsMiR 167m & Oryza sativa & ugaagcugccagcaugaucug & 21 & 100 & CF322848 & 567 & + & 5 ' & 129 & 149 & 129 & 228 \\
\hline & OsMiR 167m & & & & & CF309326 & 552 & + & 5 ' & 102 & 122 & 102 & 201 \\
\hline & OsMiR 167n & & & & 90 & BX928793 & 1039 & + & 5 ' & 277 & 297 & 277 & 366 \\
\hline & PcMiR 167 & Phaseolus coccineus & ugaagcugccagcaugaucuu & 21 & 69 & CA916400 & 283 & + & 5 ' & 135 & 155 & 135 & 203 \\
\hline & PptMiR 167 & Populus tremula $x$ Populus tremuloides & ugaagcugccagcaugaucug & 21 & 409 & BU810017 & 684 & + & 5 ' & 256 & 276 & 256 & 664 \\
\hline & SoMiR 167a & Saccharum officinarum & ugaagcugecagcaugaucug & 21 & 149 & CA284394 & 729 & - & $3^{\prime}$ & 629 & 649 & 501 & 649 \\
\hline & SoMiR 167a & & & & & CA284319 & 659 & + & 5 ' & 123 & 143 & 123 & 271 \\
\hline & SoMiR $167 \mathrm{~b}$ & & & & 139 & CA287325 & 456 & + & 5 ' & 136 & 156 & 136 & 274 \\
\hline & SMiR 167 & Saccharum sp & ugaagcugccagcaugaucug & 21 & 139 & CF571260 & 403 & + & 5 ' & 144 & 164 & 144 & 282 \\
\hline & TaMiR 167 & Triticum aestivum & ugaagcugacagcaugaucua & 21 & 210 & CK209889 & 1031 & + & $3^{\prime}$ & 397 & 417 & 208 & 417 \\
\hline & ZmMiR $167 \mathrm{~m}$ & Zea mays & ugaagcugccagcaugaucug & 21 & 80 & CF630597 & 417 & - & 3 ' & 365 & 385 & 306 & 385 \\
\hline 168 & AtMiR 168m & Arabidopsis thaliana & ucgcuuggugcaggucgggaa & 21 & 104 & H77158 & 464 & + & 5 ' & 42 & 62 & 42 & 145 \\
\hline & GmMiR 168m & Glycine max & ucgcuuggugcaggucgggaa & 21 & 88 & BE661028 & 688 & + & 5 ' & 272 & 292 & 272 & 359 \\
\hline & GmMiR 168m & & & & & AW424354 & 195 & + & 5 ' & 20 & 40 & 20 & 107 \\
\hline & GmMiR $168 \mathrm{~m}$ & & & & & BE659175 & 300 & - & $3^{\prime}$ & 254 & 274 & 187 & 274 \\
\hline & HtMiR 168 & Hedyotis terminalis & ucgcuuggugcaggucgggaa & 21 & 140 & СВ076866 & 639 & + & 5 ' & 168 & 188 & 168 & 307 \\
\hline & HvMiR 168 & Hordeum vulgare subsp. vulgare & ucgcuuggugcagaucgggac & 21 & 66 & CA003609 & 456 & + & 5 ' & 127 & 147 & 127 & 192 \\
\hline & HvMiR 168 & & & & & & 418 & + & 5 ' & 128 & 148 & 128 & 193 \\
\hline & LeMiR 168 & Lycopersicon esculentum & ucgcuuggugcaggucgggac & 21 & 145 & BF097936 & 572 & + & 5 ' & 16 & 36 & 16 & 160 \\
\hline & LeMiR 168 & & & & & BE461111 & 551 & + & 5 ' & 16 & 36 & 16 & 160 \\
\hline & LeMiR 168 & & & & & BE461110 & 572 & + & 5 ' & 16 & 36 & 16 & 160 \\
\hline & OsMiR 168m & Oryza sativa & ucgcuuggugcagaucgggac & 21 & 67 & CB667935 & 768 & + & 5 ' & 105 & 125 & 105 & 171 \\
\hline & OsMiR 168m & & & & & CB662543 & 804 & + & 5 ' & 113 & 133 & 113 & 179 \\
\hline & OsMiR 168m & & & & & CB655478 & 698 & + & 5 ' & 116 & 136 & 116 & 182 \\
\hline & OsMiR 168m & & & & & CB629782 & 733 & + & 5 ' & 12 & 32 & 12 & 78 \\
\hline & OsMiR 168m & & & & & CA756101 & 607 & + & 5 , & 118 & 138 & 118 & 184 \\
\hline & PbtMiR 168 & $\begin{array}{l}\text { Populus balsamifera subsp. } \\
\text { trichocarpa }\end{array}$ & ucgcuuggugcaggucgggaa & 21 & 118 & BU871538 & 508 & + & $5^{\prime}$ & 37 & 57 & 37 & 154 \\
\hline & PLMiR 168 & Populus tremula & ucgcuuggugcaggucgggaa & 21 & 118 & BU893331 & 297 & + & 5 ' & 102 & 122 & 102 & 219 \\
\hline & PLMiR 168 & & & & & BU889659 & 436 & + & 5 ' & 109 & 129 & 109 & 226 \\
\hline & PtMiR 168 & & & & & BU888204 & 520 & + & 5 ' & 103 & 123 & 103 & 220 \\
\hline & PptMiR 168a & Populus tremula $x$ Populus tremuloides & ucgcuuggugcaggucgggaa & 21 & 118 & BU811772 & 503 & + & 5 ' & 53 & 73 & 53 & 170 \\
\hline & PptMiR 168a & & & & & BU809183 & 461 & + & 5 ' & 109 & 129 & 109 & 226 \\
\hline & PptMiR 168b & & & & 140 & BU886509 & 514 & + & 5 ' & 70 & 90 & 70 & 209 \\
\hline & SsMiR 168 & Saccharum sp & ucgcuuggugcagaucgggac & 21 & 65 & CF576659 & 636 & + & 5 ' & 193 & 213 & 193 & 257 \\
\hline & SoMiR 168 & Saccharum officinarum & ucgcuuggugcagaucgggac & 21 & 65 & CA173899 & 663 & + & 5 ' & 164 & 184 & 164 & 227 \\
\hline & SoMiR 168 & & & & & CA156827 & 708 & + & 5 ' & 152 & 172 & 152 & 216 \\
\hline & SoMiR 168 & & & & & CA123434 & 622 & + & 5 ' & 184 & 204 & 184 & 248 \\
\hline & StMiR 168 & Solamum tuberosum & ucgcuuggugcaggucgggac & 21 & 133 & CK254942 & 865 & + & 5 ' & 85 & 105 & 85 & 217 \\
\hline & StMiR 168 & & & & & CK249947 & 916 & + & 5 ' & 12 & 32 & 12 & 144 \\
\hline & StMiR 168 & & & & & CK246126 & 980 & + & 5 ' & 12 & 32 & 12 & 144 \\
\hline & StMiR 168 & & & & & CK243936 & 770 & + & 5 ' & 10 & 30 & 10 & 142 \\
\hline & SbMiR 168 & Sorghum bicolor (sorghum) & ucgcuuggugcagaucgggac & 21 & 67 & $\mathrm{CN} 133168$ & 669 & + & 5 ' & 173 & 193 & 173 & 239 \\
\hline & SbMiR 168 & & & & & CD213423 & 572 & + & 5 ' & 173 & 193 & 173 & 239 \\
\hline & SbMiR 168 & & & & & CD210663 & 530 & + & 5 ' & 183 & 203 & 183 & 249 \\
\hline & SbMiR 168 & & & & & CD209437 & 662 & + & 5 ' & 184 & 204 & 184 & 250 \\
\hline & SbMiR 168 & & & & & CD209338 & 549 & + & 5 ' & 179 & 199 & 179 & 245 \\
\hline & SbMiR 168 & & & & & CD207772 & 531 & + & 5 ' & 193 & 213 & 193 & 259 \\
\hline & SbMiR 168 & & & & & CD205823 & 586 & + & 5 ' & 182 & 202 & 182 & 248 \\
\hline & SbMiR 168 & & & & & CD205059 & 581 & + & 5 , & 182 & 202 & 182 & 248 \\
\hline & SbMiR 168 & & & & & CN145907 & 312 & + & 5 ' & 95 & 115 & 95 & 161 \\
\hline & VvMiR 168 & Vitis vinifera & ucgcuuggugcaggucgggaa & 21 & 110 & CF604588 & 521 & + & 5 & 52 & 72 & 52 & 161 \\
\hline & ZmMiR 168m & Zea mays & ucgcuuggugcagaucgggac & 21 & 65 & BU099153 & 532 & + & 5 & 62 & 82 & 62 & 126 \\
\hline & ZmMiR $168 \mathrm{~m}$ & & & & & BG842539 & 498 & + & 5 , & 97 & 117 & 97 & 161 \\
\hline & ZmMiR $168 \mathrm{~m}$ & & & & & CA826777 & 522 & + & 5 ' & 62 & 82 & 62 & 126 \\
\hline & ZmMiR $168 \mathrm{~m}$ & & & & & BG842528 & 494 & + & 5 ' & 97 & 117 & 97 & 161 \\
\hline 169 & GmMiR $169 \mathrm{~m}$ & Glycine $\max$ & cagccaaggaugacuugccga & 21 & 98 & BF595231 & 457 & + & $3^{\prime}$ & 386 & 406 & 309 & 406 \\
\hline
\end{tabular}


Identifying new plant microRNAs using EST analysis

Tab. 3 New miRNAs identified from plants (continued-5)

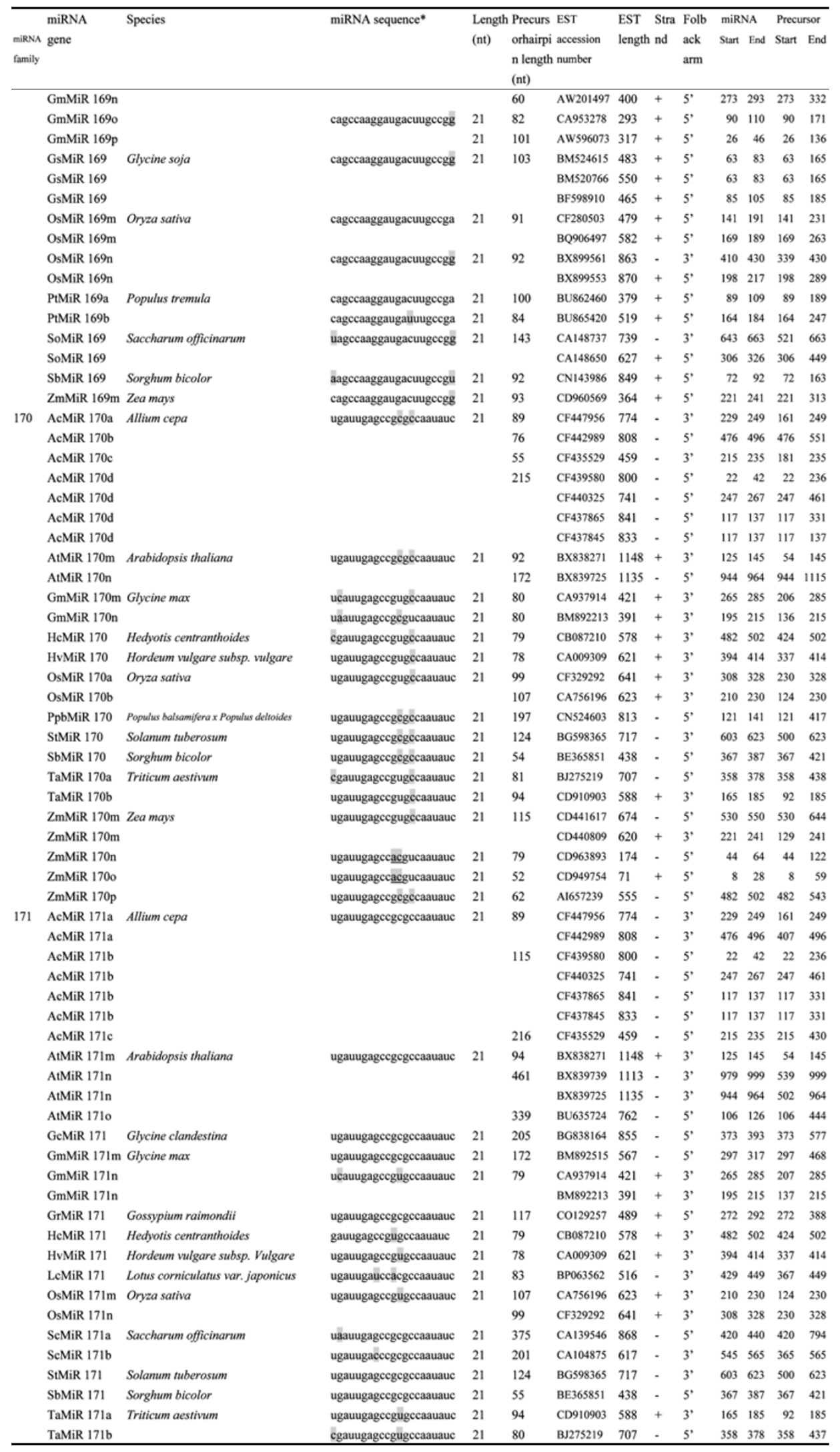


Tab. 3 New miRNAs identified from plants (continued-6)

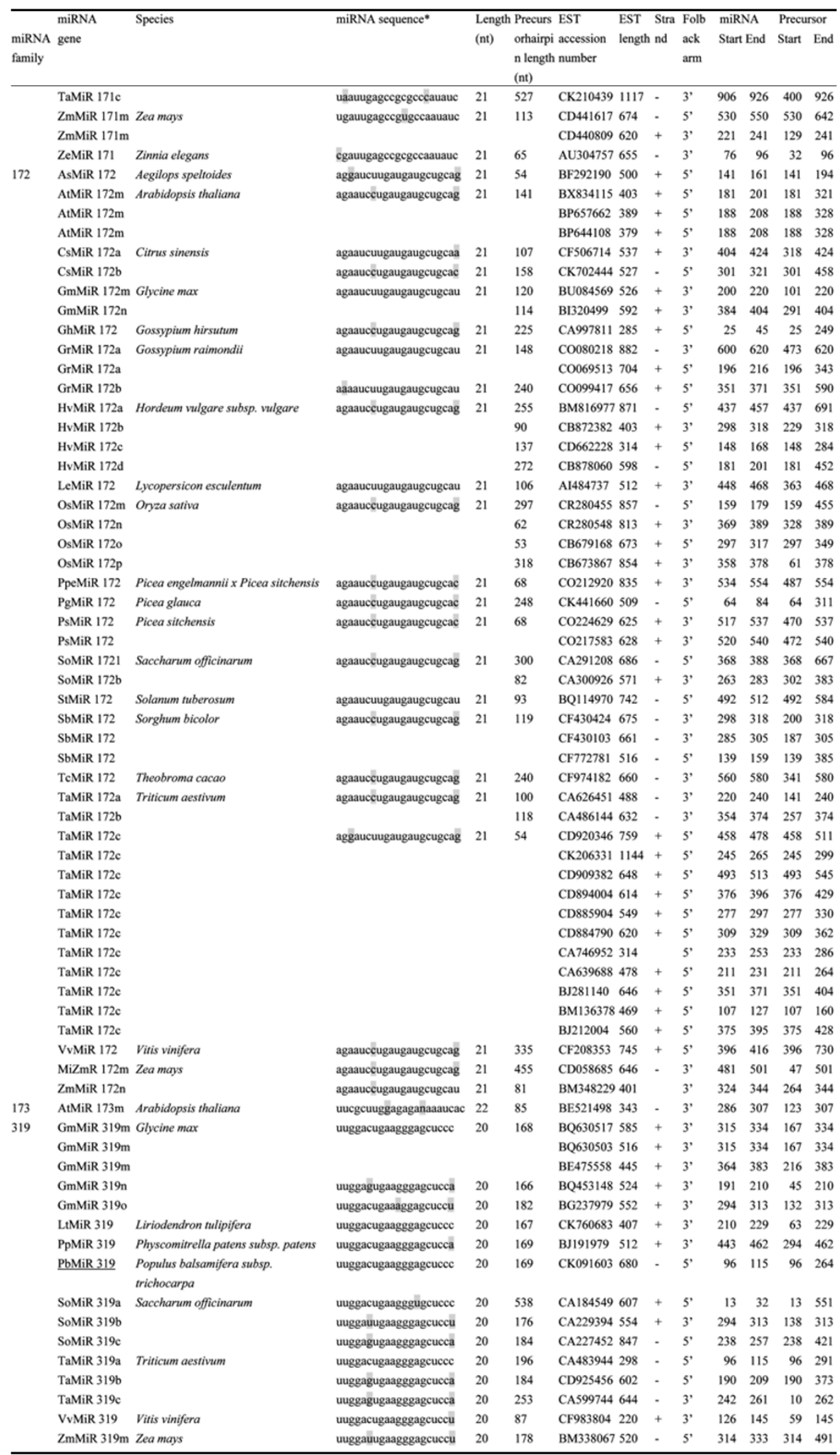

* Letters in shadow indicate that the nucleotides are different from the previously known Arabidopsis miRNAs 
Identifying new plant microRNAs using EST analysis

Tab. 4 ESTs matched by known Arabidopsis miRNAs, and identified as new miRNAs in plants

\begin{tabular}{|c|c|c|c|c|}
\hline Plant species & EST number in dbEST* & $\begin{array}{l}\text { EST contigs identified } \\
\text { with known miRNAs }\end{array}$ & Identified $\%$ & miRNA \\
\hline Aegilops speltoides & 4,315 & 1 & 0.023 & 1 \\
\hline Allium сера & 19,582 & 14 & 0.071 & 7 \\
\hline Arabidopsis thaliana & 322,641 & 20 & 0.006 & 17 \\
\hline Brassica napus & 37,159 & 1 & 0.003 & 1 \\
\hline Capsicum annuum & 22,961 & 3 & 0.013 & 3 \\
\hline Citrus sinensis & 45,192 & 5 & 0.011 & 5 \\
\hline Cycas rumphii & 5,952 & 1 & 0.017 & 1 \\
\hline Eschscholzia californica & 8,531 & 1 & 0.012 & 1 \\
\hline Glycine clandestina & 933 & 1 & 0.107 & 1 \\
\hline Glycine $\max$ & 334,668 & 55 & 0.016 & 45 \\
\hline Glycine soja & 16,508 & 3 & 0.018 & 1 \\
\hline Gossypium arboreum & 39,007 & 2 & 0.005 & 2 \\
\hline Gossypium hirsutum & 14,650 & 1 & 0.007 & 1 \\
\hline Gossypium raimondii & 63,577 & 12 & 0.018 & 9 \\
\hline Hedyotis centranthoides & 5,416 & 3 & 0.055 & 3 \\
\hline Hedyotis terminalis & 4,875 & 1 & 0.021 & 1 \\
\hline Helianthus annuus & 59,841 & 3 & 0.005 & 3 \\
\hline Hordeum vulgare & 356,856 & 27 & 0.008 & 17 \\
\hline Ipomoea batatas & 4,168 & 1 & 0.024 & 1 \\
\hline Ipomoea nil & 25,899 & 10 & 0.037 & 5 \\
\hline Lactuca sativa & 68,188 & 3 & 0.004 & 3 \\
\hline Liriodendron tulipifera & 4,282 & 2 & 0.047 & 2 \\
\hline Lotus corniculatus var. japonicus & 111,455 & 6 & 0.006 & 5 \\
\hline Lupinus luteus & 364 & 1 & 0.275 & 1 \\
\hline Lycopersicon esculentum & 150,596 & 13 & 0.009 & 9 \\
\hline Malus $x$ domestica (apple tree) & 129,134 & 3 & 0.002 & 3 \\
\hline Medicago truncatula & 187,763 & 9 & 0.005 & 8 \\
\hline Nicotiana benthamiana & 26,924 & 5 & 0.019 & 4 \\
\hline Nicotiana tabacum & 10,945 & 1 & 0.009 & 1 \\
\hline Nuphar advena & 6,854 & 3 & 0.044 & 3 \\
\hline Oryza sativa & 284,007 & 41 & 0.0144 & 25 \\
\hline Pennisetum glaucum & 2,528 & 1 & 0.040 & 1 \\
\hline Persea americana & 4,276 & 1 & 0.023 & 1 \\
\hline Phaseolus coccineus & 20,120 & 2 & 0.010 & 2 \\
\hline Physcomitrella patens & 82,420 & 2 & 0.002 & 2 \\
\hline Picea engelmannii $x$ Picea sitchensis & 12,125 & 1 & 0.008 & 1 \\
\hline Picea glauca & 55,108 & 6 & 0.011 & 6 \\
\hline Picea sitchensis & 12,065 & 4 & 0.033 & 3 \\
\hline Pinus taeda & 152,514 & 13 & 0.009 & 5 \\
\hline Populus balsamifera subsp. trichocarpa & 26,825 & 3 & 0.011 & 3 \\
\hline Populus balsamifera $x$ Populus deltoides & 16,431 & 3 & 0.018 & 3 \\
\hline Populus tremula & 31,288 & 6 & 0.019 & 4 \\
\hline Populus tremula $x$ Populus tremuloides & 65,981 & 9 & 0.014 & 8 \\
\hline Prunus armeniaca & 5,735 & 2 & 0.035 & 2 \\
\hline Saccharum officinarum & 246,301 & 34 & 0.014 & 25 \\
\hline Saccharum sp & 9,636 & 2 & 0.021 & 2 \\
\hline Schedonorus arundinaceus & 2,462 & 2 & 0.081 & 1 \\
\hline Secale cereale & 9,194 & 1 & 0.011 & 1 \\
\hline Sesamum indicum & 3,328 & 2 & 0.060 & 2 \\
\hline Solanum tuberosum & 158,154 & 17 & 0.011 & 13 \\
\hline Sorghum bicolor & 190,946 & 30 & 0.016 & 14 \\
\hline Sorghum propinquum & 21,387 & 2 & 0.009 & 2 \\
\hline Theobroma cacao & 6,557 & 1 & 0.015 & 1 \\
\hline Triticum aestivum & 559,149 & 32 & 0.006 & 19 \\
\hline Triticum turgidum & 8,714 & 1 & 0.011 & 1 \\
\hline Vitis vinifera & 141,616 & 8 & 0.006 & 7 \\
\hline Zea mays & 415,211 & 36 & 0.009 & 23 \\
\hline Zinnia elegans & 17,529 & 3 & 0.017 & 3 \\
\hline Total & $4,650,843$ & 476 & 0.010 & 344 \\
\hline
\end{tabular}

* dbEST release 071604: Summary by Organism - July 16, 2004 
A

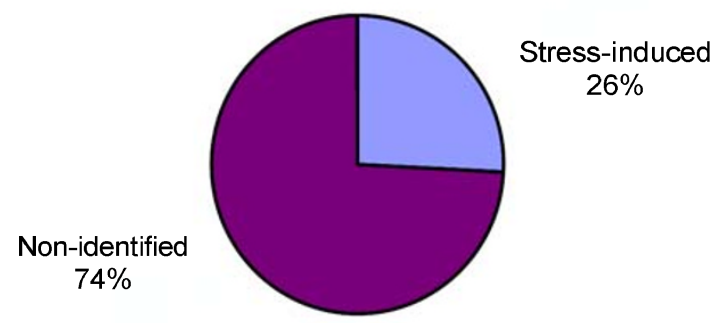

B

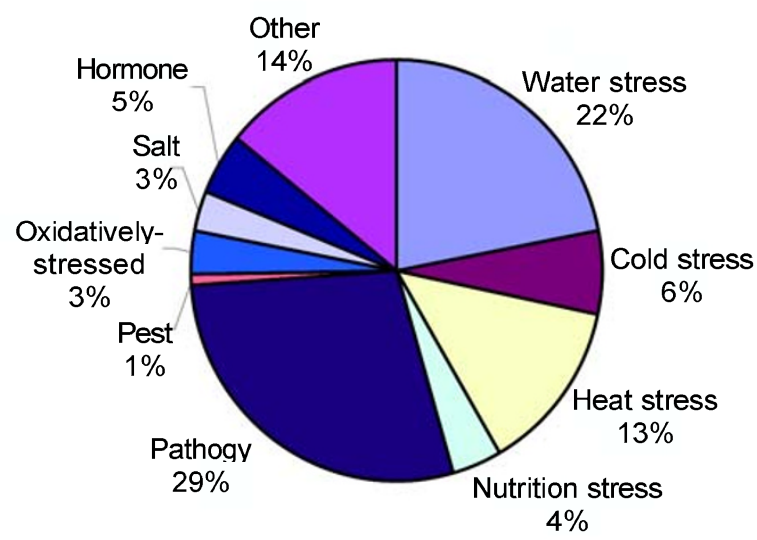

Fig. 4 miRNAs are induced and regulated by environmental biotic and abiotic stresses. (A) 26\% of ESTs were obtained from stressinduced tisssues. (B) ESTs containing miRNAs were obtained from tissue by various biotic and abiotic environmental stresses.
Out of the 476 identified EST contigs containing miRNAs, 123 , or $25.84 \%$, were found in stress-induced plant tissues (Fig. 4A). These environmental stresses included abiotic and biotic stresses, such as drought, heat, cold, salinity, pathogen infection, or pests. In the 123 EST contigs, $36(29 \%)$ EST contigs were associated with pathogen infection; 28 (22\%) were associated with water stress; and $25(19 \%)$ were associated with temperature stress. Other stresses, such as nutrition deficiency, salinity, and oxidative stress produced $4 \%, 3 \%$, and $3 \%$ EST contigs, respectively (Fig. 4B). Abscisic acid (ABA) is considered a plant stress hormone, and jasmonic acid (JA) and salicylic acid (SA) are believed to be related to plant response to environmental stress. In this study, we found that 6 of the 123 EST contigs were created from the tissue induced by ABA, JA, SA, or other hormones. The other $18(14 \%)$ EST contigs were created from the tissues induced by other abiotic and biotic environmental stresses, such as darkness or oxygen deficiency, wound treatment, acidor alkaline-treatment.

Among all of the plant ESTs included in the TIGR database, $23 \%$ were from different stressed tissues. Here we found that $25.84 \%$ of total ESTs containing potential miRNA were obtained from stress-induced plant tissues. Does this really mean that these microRNAs were induced by stress? More experiments need to be done to confirm this. However, recently three reports have confirmed our

Tab. 5 Tissue-specific expression of plant miRNAs

\begin{tabular}{|c|c|c|c|c|c|c|c|c|c|c|c|c|c|c|c|}
\hline$\overline{\text { miRNAs }}$ & Flower & Leaf & Root & $\begin{array}{c}\text { Whole } \\
\text { seedling }\end{array}$ & $\begin{array}{l}\text { Adult } \\
\text { plant }\end{array}$ & $\begin{array}{c}\text { Developing } \\
\text { embryos or } \\
\text { seeds }\end{array}$ & $\begin{array}{l}\text { Mixed } \\
\text { tissue }\end{array}$ & Stem & $\begin{array}{c}\text { Shoot } \\
\text { meristem }\end{array}$ & $\begin{array}{c}\text { Fruit, } \\
\text { silique } \\
\text { and } \\
\text { spike }\end{array}$ & Callus & $\begin{array}{l}\text { Somatic } \\
\text { embryos }\end{array}$ & $\begin{array}{l}\text { Xylem } \\
\text { and } \\
\text { phloem }\end{array}$ & Fiber & Total \\
\hline 156 & 18 & 20 & 8 & 2 & 2 & 11 & 4 & 6 & 13 & 7 & 2 & 1 & 1 & & 92 \\
\hline 157 & 20 & 5 & 3 & 5 & 1 & 3 & 5 & 3 & 1 & 5 & 2 & 1 & & & 52 \\
\hline 158 & 2 & & & & & & 1 & & & & & & & 1 & 4 \\
\hline 159 & 9 & 6 & 4 & 5 & & & & 2 & 5 & & 3 & & & & 34 \\
\hline 160 & 4 & 10 & 1 & 1 & & 3 & 2 & 1 & & & 4 & & & & 22 \\
\hline 161 & & & & & & & & & & & & & & & \\
\hline 162 & 2 & 1 & 1 & 2 & & & & 1 & & 1 & & & & & 8 \\
\hline 163 & 1 & 1 & 10 & & & & & & 1 & & & & 1 & 1 & 14 \\
\hline 164 & 2 & & 1 & & & 3 & & & & 1 & & & & & 8 \\
\hline 165 & 1 & 1 & 1 & & & & & & & & & & & & 2 \\
\hline 166 & 2 & 3 & 7 & 5 & & & 3 & 1 & 1 & & & 1 & 1 & & 24 \\
\hline 167 & 1 & 3 & 1 & 3 & & 5 & 1 & 2 & & 1 & 1 & & & & 18 \\
\hline 168 & 4 & 12 & 6 & 9 & & 3 & 1 & 3 & & & 4 & & & & 41 \\
\hline 169 & & 7 & & 3 & & 2 & & 2 & & & & & & & 14 \\
\hline 170 & 3 & 4 & 1 & & & 4 & 8 & & 1 & 1 & 1 & & & & 23 \\
\hline 171 & 3 & 7 & 2 & 1 & & 4 & 10 & 3 & & 2 & 1 & & & & 31 \\
\hline 172 & 9 & 12 & 7 & 6 & & 5 & 2 & 1 & 2 & 3 & & & & & 45 \\
\hline 173 & & & & & & 1 & & & & & & & & & 1 \\
\hline 319 & 6 & 3 & 1 & 1 & & 1 & 1 & 3 & & 1 & & & & & 16 \\
\hline Total & 87 & 95 & 54 & 43 & 3 & 45 & 38 & 28 & 24 & 22 & 18 & 3 & 3 & 2 & 449 \\
\hline
\end{tabular}


hypothesis. miRNA 159, 319, 395 and 402 were regulated by GA, cold, water and sulfate starvation stress, individually $[11,14,37]$.

Expression of plant miRNAs appears to be developmental or tissue specific. miRNAs are somewhat more strongly expressed in seedlings than in adult plants. Out of the 449 identified EST contigs which contain miRNAs and indicate origin tissues, 43 ESTs were obtained from young seedlings, but only 3 from adult plants (Tab. 5). Early and rapid growth stages may need more miRNA to regulate their gene expression. This is confirmed by recent reports $[11,23,34]$. In previous investigations, most miRNAs have been found to be strongly expressed in floral and leaf tissues $[7,11,34,38-41]$. This observation was also supported by this study. Out of the 338 newly identified miRNAs in 60 different plant species, the majority were found in flowers and leaves. Fewer were found in roots, developing embryos, seeds or other tissues (Fig. 5 and Tab. 5). This indicates that miRNAs play important roles in plant growth and development other than controlling leaf and floral development [7, 11, 34, 38-41]. However, different miRNAs have different expression patterns. miRNA 157 preferentially expresses in flowers, but miRNAs 160, 163, and 168 preferentially express in leaves rather than in other tissues. miRNAs 156,159 , and 172 preferentially express in both flowers and leaves. miRNA 163 preferentially ex- presses in roots. Although we obtained EST contigs containing miRNAs from other tissues, it did not appear that these miRNAs preferentially express in those tissues (Tab. 5). However, they may play other roles in those tissues in regulating expression of certain genes. In this study, miRNAs also have been found to be expressed in other tissues, such as roots, vascular tissues, stems, callus, somatic embryoes and fibers. This suggests that miRNAs play some role and regulate certain gene expression in these tissues.

In the EST database, ESTs were unequally obtained from different tissues. Some tissues may contribute more ESTs than other tissues. Thus, more experiments need to be conducted to confirm this conclusion. However, this approach gave us more clues to study plant microRNAs, and this strategy alleviates the usually difficult step of predicating the correct tissues and conditions to search for expression evidence in a directed manner.

\section{DISCUSSION}

miRNAs are widespread and highly conserved in the plant kingdom, and they may have the same ancestor in very early evolution

miRNAs are widespread in plant species and eukaryotes. In previous studies, 71 miRNAs have been reported in Arabidopsis and rice. Out of the 71 miRNAs, 43 were

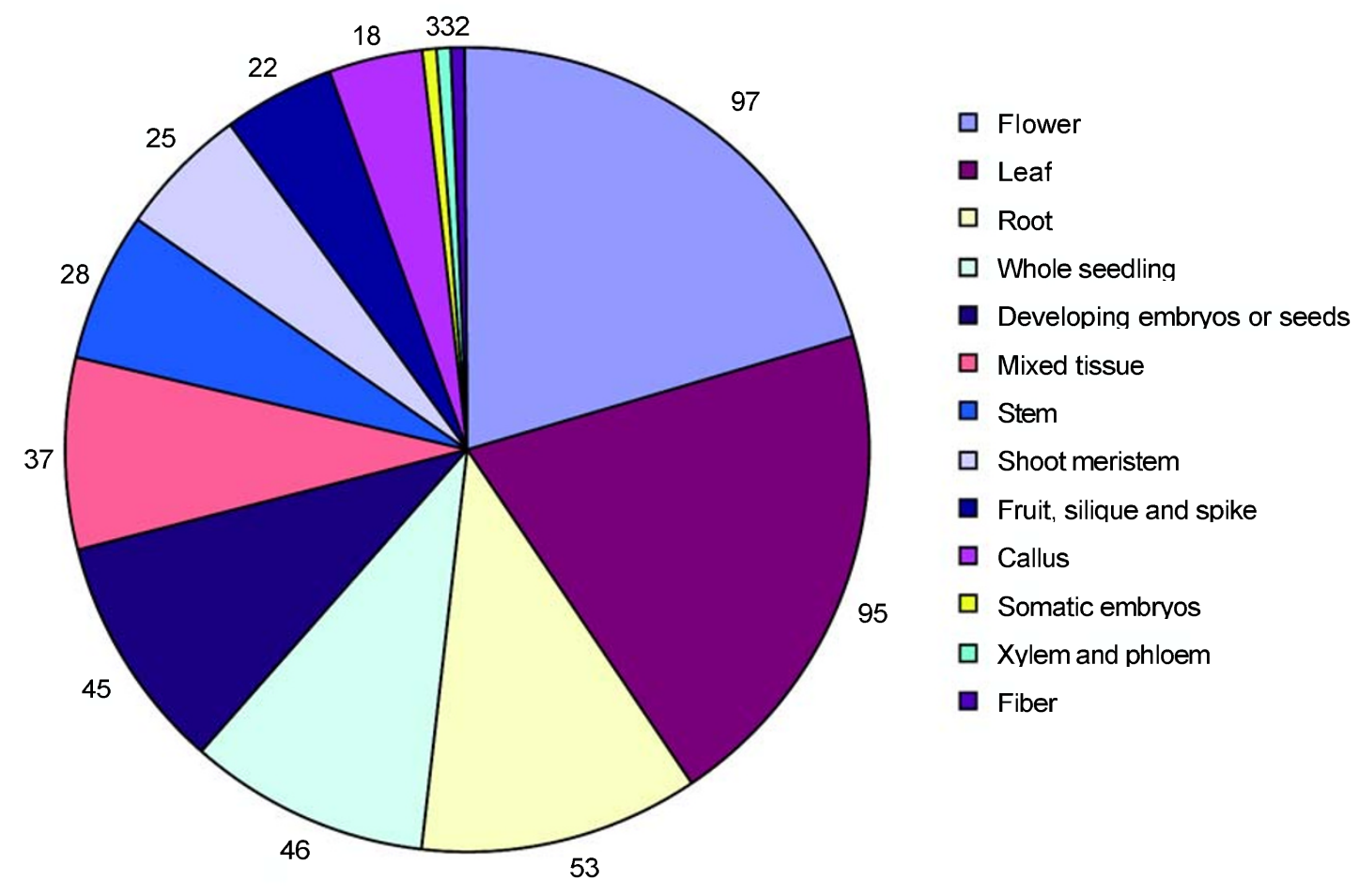

Fig. 5 Preferential expression of miRNAs 
predicted and identified in Arabidopsis, and 28 in rice (the miRNA Registry database, Release 3.1, April 2004). In this study, we found 338 new miRNAs in 60 plant species using EST analysis and BLAST algorithms. With improvements in the strategy and technology for identifying miRNAs, more miRNAs will be discovered. In metazoans, such as Drosophila and humans, computational strategy suggests that miRNAs constitute nearly $1 \%$ of predicated proteincoding genes. In comparison with animals, a distinguishing feature of plants is that they are sessile and thus have to cope with, rather than move to avoid, complicatedly adverse environments and invasion of animals [47]. Plants should therefore have more complicated mechanisms to control gene expression and gene regulation. If miRNAs are involved in these mechanisms, there should be more miRNAs in plant genomes than in animal genomes. Arabidopsis is a model plant species for genome research; its genome sequence was published in 2000 [42]. The Arabidopsis Information Resource (TAIR) shows that Arabidopsis has currently 21,316 proteins (http://www. arabidopsis.org/tools/bulk/protein/index.jsp) excluding proteins of unknown subcellular location and chloroplastand mitochondrion- targeted proteins. If plants have the same $1 \%$ miRNAs in their genome, Arabidopsis should have 213 miRNA genes. However, only 43 miRNAs have been reported in previous studies, plus the 17 newly discovered miRNAs candidates in this report; 60 miRNAs have been discovered in Arabidopsis. This is far from 1. $0 \%$ of the protein-coding genes, so more miRNAs still await discovery in plants.

miRNAs are highly conserved among different plant species, including dicots and monocots. There is no nucleotide base substitution in the majority of miRNA families from different plant species. Some species only have a few (0-2) nucleotide base substitutions with Arabidopsis miRNAs. This suggests miRNAs may have the same ancestor in early evolution. However, some microRNAs may have a very high rate of divergence even if some are highly conserved.

\section{Stress may play an important role in miRNAs regulation}

It is a complicated mechanism for both animals and plants to respond to stress. In this specific response, miRNA may be involved. In animals, loss of function of miR 14 increased sensitivity of fly to different stresses [43], and nutrient stress may induce expression of miRNA 234 [19]. In this study, we found $26 \%$ of EST contigs containing miRNAs are related to different biotic or abiotic environmental stresses, and out of these ESTs, 22\% related to water stress, $19 \%$ related to temperature stress, and $29 \%$ related to pathogen infection (Fig. 4). These data suggest that environmental stress may play an important role in miRNA gene expression in plants. This conclusion is also supported by recent reports [11, 14, 37]. JonesRhoades and Bartel (2004) showed that sulfate starvation induced overexpression of miRNA 395 [14]. The experiment by Sunkar and Zhu (2004) showed that miRNA 402 is strongly overexpressed under the condition of dehydration, cold, salt stress, or ABA treatment [11]. However, miRNA 319 is induced by cold [11], and miRNA 159 is regulated by GA [37].

Biotic and abiotic stress may function as signals to control and to regulate miRNA genes in plants (Fig. 6). First, these biotic or abiotic stressors form different signaling in plant cells, then induce a $\mathrm{Ca}^{2+}$ signal or MAPK signal which interacts with a transcriptional factor, and produces down or over expression of the targeted miRNA genes (Fig. 6). The study of specific responses of miRNAs to environmental stress will help us improve plant resistance to environmental stresses, especially disease, drought, and salinity stress.

Another function of miRNAs may be involved in signal transduction. In this study, we found 7 ESTs containing miRNAs are associated with ABA or other hormone signaling. After we searched the NSF 2010 Arabidopsis Small RNA Project database (http://cgrb.orst.edu/ smallRNA/) of small known RNAs and compared all previous known Arabidopsis miRNA to ABA signaling related genes with BLAST algorithms, we found miRNAs may be involved in the regulation of the ABA signaling genes.

Although several studies have tried to determine what produces microRNAs, little is known regarding miRNA biogenesis in plants. The data in this study suggest that environmental stresses and developmental switching may function as signaling to induce pri-miRNA transcription by RNA polymerases (Fig. 6). After pri-miRNAs are synthesized, they are processed to miRNA precursors or pre-miRNAs by the double-stranded RNase DICER-LIKE (DCL) $[7,34,41]$. Finally, the cleaved pre-miRNA will be translocated into cytoplasm by a HASTY protein, and cleaved into mature miRNAs (Fig. 6). In the cytosol, miRNA regulate gene expression at posttranscriptional levels by miRNA cleavage or by translational repression [2]. In animals, both of the two regulatory mechanisms are common on repression of target gene expression [2]. In plants, the majority of miRNAs interact with the internal regions of target mRNAs through perfect or near perfect basepairing to cleave the mRNAs $[1,39,44]$. However, for some miRNAs, such as miRNA 172, although they have perfect or near-perfect complimentarity sequence with target mRNAs, they inhibit the expression of target genes by binding to a unique site located within the open reading frame of the target mRNAs rather than miRNA cleavage $[38,45]$. 


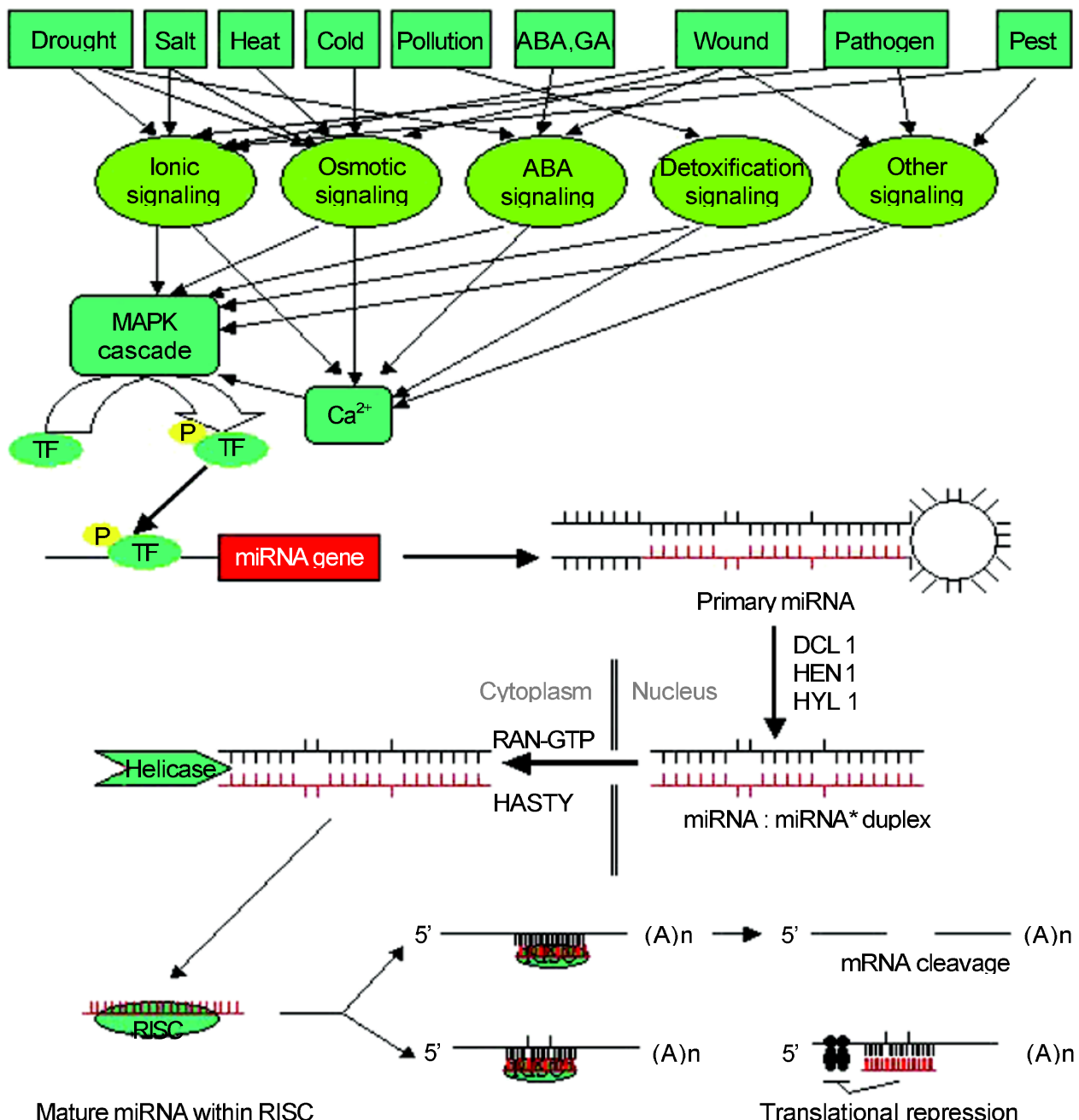

Fig. 6 A model for miRNA biogenesis, regulation of miRNA gene regulation, and the pathway of miRNA function.

\section{False positives}

Existence of false positives is one problem in many studies. To reduce the number of false positives, we combined multiple methods in this study. This is similar to that described by Bonnet et al. [13]. First, the number of EST hits is dramatically reduced after comparing EST sequences to previously known Arabidopsis miRNA sequences. According to the characteristic that plant miRNAs are highly conserved and only a few nucleotides change between plant species, ESTs with only 0-2 mismatched nucleotides with previously known Arabidopsis miRNA were considered as potential miRNA candidates. Second, potential ESTs were reduced about $50 \%$ by considering the secondary structure parameters based on previous reports $[1,2,11$,
$13,34,46]$. Third, the number of miRNAs was reduced by considering potential miRNA target genes in the DNA database. Fourth, repeated ESTs were removed by comparing similar EST sequences. Fifth, to avoid a miscount of the total EST sequences, ESTs with n-2, n-3, n-4, and $\mathrm{n}-5$ mismatched nucleotides with previously known miRNA were chosen and paired with known miRNA sequences. Sixth, the matched ESTs were blasted against a database of known proteins to kick out the potential ESTs which actually code a protein rather than a structural RNA. After these six steps and other combined strategies, the number of EST hits dramatically reduced from 18,694 to 384 . This gave us more confidence in identifying miRNAs.

If these identified miRNAs are confirmed by experi- 
mental evidence, such as northern blotting, it will give us more confidence in the newly identified miRNAs and the hypothesis that stress may play an important role in miRNA regulation. However, ESTs are the true products of gene expression, and in some case they may be considered as the result of northern blotting. Actually, Smalheiser [36] successfully predicted the existence of a population of chimeric microRNA precursor-mRNA transcripts expressed in normal human and mouse tissues only by EST analysis. Bonnet et al. [13] used the EST database to confirm their 91 potential Arabidopsis and rice miRNAs identified by a computational strategy, and several genes have been identified by only EST analysis [27-29]. Thus, we have confidence in these identified miRNAs by EST analysis. EST analysis also gave us more information on miRNAs in other plant species in which it was impossible to identify miRNAs by traditional computational strategy due to lack of a DNA sequence. Finally, EST analysis provides information for designing more new experiments to understand miRNAs [36].

Received, Mar 1, 2005

Revised, Mar 28, 2005

Accepted, Apr 4, 2005

\section{REFERENCES}

1 Bartel B, Bartel DP. MicroRNAs: at the root of plant development? Plant Physiol 2003; 132:709-17.

2 Bartel DP. MicroRNAs: Genomics, biogenesis, mechanism, and function. Cell 2004; 116:281-97.

3 Mallory AC, Vaucheret H. MicroRNAs: something important between the genes. Curr Opin Plant Biol 2004; 7:120-5.

4 Carrington JC, Ambros V. Role of microRNAs in plant and animal development. Science 2003; 301:336-8.

5 Hunter C, Poethig RS. Missing links: miRNAs and plant development. Curr Opin Genet Dev 2003; 13:372-8.

6 Griffiths-Jones S. The microRNA Registry. Nucleic Acids Res 2004; 32:D109-11.

7 Juarez MT, Kui JS, Thomas J, Heller BA, Timmermans MCP. microRNA-mediated repression of rolled leafl specifies maize leaf polarity. Nature 2004; 428:84-8.

8 McHale NA, Koning RE. MicroRNA-directed cleavage of Nicotiana sylvestris PHAVOLUTA mRNA regulates the vascular cambium and structure of apical meristems. Plant Cell 2004; 16: 1730-40.

9 Lai EC. microRNAs: Runts of the genome assert themselves. Curr Biol 2003; 13:R925-36.

10 Lai EC, Tomancak P, Williams RW, Rubin GM. Computational identification of Drosophila microRNA genes. Genome Biol 2003; 4:R42.

11 Sunkar R, Zhu JK. Novel and stress-regulated microRNAs and other small RNAs from Arabidopsis. Plant Cell 2004; 16:200119.

12 Adai A, Johnson C, Mlotshwa S, et al. Computational prediction of miRNAs in Arabidopsis thaliana. Genome Res 2005; 15: 78-91.
13 Bonnet E, Wuyts J, Rouze P, Van de Peer Y. Detection of 91 potential in plant conserved plant microRNAs in Arabidopsis thaliana and Oryza sativa identifies important target genes. Proc Natl Acad Sci U S A 2004; 101:11511-6.

14 Jones-Rhoades MW, Bartel DP. Computational identification of plant MicroRNAs and their targets, including a stress-induced miRNA. Mol Cell 2004; 14:787-99.

15 Lai EC. Predicting and validating microRNA targets. Genome Biol 2004; 5:A115.

$16 \mathrm{Li} \mathrm{Y,} \mathrm{Li} \mathrm{W,} \mathrm{Jin} \mathrm{YX.} \mathrm{Computational} \mathrm{identification} \mathrm{of} \mathrm{novel} \mathrm{family}$ members of microRNA genes in Arabidopsis thaliana and Oryza sativa. Acta Biochim Biophys Sin 2005; 37:75-87.

17 Rhoades MW, Reinhart BJ, Lim LP, et al. Prediction of plant microRNA targets. Cell 2002; 110:513-20.

18 Lim LP, Glasner ME, Yekta S, Burge CB, Bartel DP. Vertebrate microRNA genes. Science 2003; 299: 1540 .

19 Lim LP, Lau NC, Weinstein EG, et al. The microRNAs of Caenorhabditis elegans. Genes Dev 2003; 17:991-1008.

20 Krol J, Krzyzosiak WJ. Structural aspects of microRNA biogenesis. IUBMB Life 2004; 56:95-100.

21 Lewis BP, Shih IH, Jones-Rhoades MW, Bartel DP, Burge CB. Prediction of mammalian microRNA targets. Cell 2003; 115:78798.

22 Rajewsky N, Socci ND. Computational identification of microRNA targets. Dev Biol 2004; 267:529-35.

23 Wang JF, Zhou H, Chen YQ, Luo QJ, Qu LH. Identification of 20 microRNAs from Oryza sativa. Nucleic Acids Res 2004; 32: 1688-95.

24 Wang XJ, Reyes JL, Chua NH, Gaasterland T. Prediction and identification of Arabidopsis thaliana microRNAs and their mRNA targets. Genome Biology 2004; 5:R65.

25 Adams MD, Kelley JM, Gocayne JD, et al. Complementary DNA sequencing: expressed sequence tags and human genome project. Science 1991; 252:1651-6.

26 Matukumalli LK, Grefenstette JJ, Sonstegard TS, van Tassell CP. EST-PAGE - managing and analyzing EST data. Bioinformatics 2004; 20:286-8.

27 Graham MA, Silverstein KAT, Cannon SB, VandenBosch KA. Computational identification and characterization of novel genes from legumes. Plant Physiol 2004; 135:1179-97.

28 Jung JD, Park HW, Hahn Y, et al. Discovery of genes for ginsenoside biosynthesis by analysis of ginseng expressed sequence tags. Plant Cell Rep 2003; 22:224-30.

29 Ohlrogge J, Benning C. Unraveling plant metabolism by EST analysis. Curr Opin Plant Biol 2000; 3:224-8.

30 Boguski MS, Lowe TM, Tolstoshev CM. dbEST - database for “expressed sequence tags". Nat Genet 1993; 4:332-3.

31 Altschul SF, Madden TL, Schäffer AA, et al. Gapped BLAST and PSI-BLAST: a new generation of protein database search programs. Nucleic Acids Res 1997; 25:3389-402.

32 Mathews DH, Sabina J, Zuker M, Turner DH. Expanded sequence dependence of thermodynamic parameters improves prediction of rna secondary structure. J Mol Biol 1999; 288: 911-40.

33 Zuker M. Mfold web server for nucleic acid folding and hybridization prediction. Nucleic Acids Res 2003; 31:3406-15.

34 Reinhart BJ, Weinstein EG, Rhoades MW, Bartel B, Bartel DP. MicroRNAs in plants. Genes Dev 2002; 16:1616-26.

35 Ambros V. MicroRNA pathways in flies and worms: Growth, 
death, fat, stress, and timing. Cell 2003; 113:673-6.

36 Smalheiser NR. EST analyses predict the existence of a population of chimeric microRNA precursor-mRNA transcripts expressed in normal human and mouse tissues. Genome Biol 2003; 4:403.

37 Achard P, Herr A, Baulcombe DC, Harberd NP. Modulation of floral development by a gibberellin-regulated microRNA. Development 2004; 131:3357-65.

38 Chen XM. A microRNA as a translational repressor of APETALA2 in Arabidopsis flower development. Science 2004; 303:2022-5.

39 Llave C, Xie ZX, Kasschau KD, Carrington JC. Cleavage of Scarecrow-like mRNA targets directed by a class of Arabidopsis miRNA. Science 2002; 297:2053-6.

40 Palatnik JF, Allen E, Wu XL, et al. Control of leaf morphogenesis by microRNAs. Nature 2003; 425:257-63.

41 Park W, Li JJ, Song RT, Messing J, Chen XM. CARPEL FACTORY, a Dicer homolog, and HEN1, a novel protein, act in
microRNA metabolism in Arabidopsis thaliana. Curr Biol 2002; 12:1484-95.

42 The Arabidopsis Genome Initiative. Analysis of the genome sequence of the flowering plant Arabidopsis thaliana. Nature 2000; 408:796-815.

43 Xu PZ, Vernooy SY, Guo M, Hay BA. The Drosophila MicroRNA mir-14 suppresses cell death and is required for normal fat metabolism. Current Biology 2003; 13:790-5.

44 Llave C. MicroRNAs: more than a role in plant development? Mol Plant Path 2004; 5:361-6.

45 Aukerman MJ, Sakai H. Regulation of flowering time and floral organ identity by a microRNA and its APETALA2-like target genes. Plant Cell 2003; 15:2730-41.

46 Bartel DP, Chen CZ. Micromanagers of gene expression: the potentially widespread influence of metazoan microRNAs. Nat Rev Genet 2004; 5:396-400.

47 Zhu JK. Salt and drought stress signal transduction in plants. Annu Rev Plant Biol 2002; 53:247-73. 